\title{
Atmospheric Flow Field Models Applicable for Aircraft Endurance Extension
}

\author{
Ricardo Bencatel $^{\mathrm{a}, *}$, João Borges de Sousa ${ }^{\mathrm{b}}$, Anouck Renée Girard ${ }^{\mathrm{a}}$ \\ ${ }^{a}$ Department of Aerospace Engineering \\ University of Michigan \\ Ann Arbor, Michigan, 48109, United States \\ ${ }^{b}$ Department of Electrical and Computer Engineering \\ University of Porto, School of Engineering \\ Porto, 4200-465, Portugal
}

\begin{abstract}
We present a survey of atmospheric flow field phenomena models. The studied models are selected for their potential use towards extended aircraft endurance. This work describes several flow field phenomena, i.e., air flow currents and flow velocity variations. In particular, we discuss wind shear, thermal updrafts, and gusts. We study several wind shear models, such as the Surface, Layer, and Ridge Wind Shear models, comparing their characteristics. We also describe and compare thermal updraft models, such as the Chimney and the Bubble Thermal models. To close, we review different gust models. Throughout this work, we studied several existing models, but we also introduce new ones and improved versions of existing ones. The Bubble Thermal, Layer Wind Shear, and the Ridge Wind Shear models are examples of the new models presented. Furthermore, we present the Chimney Thermal model improvements, which take into account the phenomenon interaction with the prevailing winds.
\end{abstract}

\footnotetext{
${ }^{*}$ Corresponding author:
}

Tel: +1 734255 7450; Fax: +1 7347639300

2060 François Xavier Bagnoud Building

1320 Beal Avenue

Ann Arbor, Michigan 48109-2140

United States

Email addresses: bencatel@umich.edu (Ricardo Bencatel), jtasso@fe.up.pt (João Borges de Sousa), anouck@umich.edu (Anouck Renée Girard) 
Keywords: Atmospheric Phenomena, Flow Field, Modeling, UAV, Flight Endurance

\section{Introduction}

This survey focuses on atmospheric flow field phenomena. In particular we describe models for thermal updrafts, wind shear and gusts. Thermal updrafts appear due to temperature differences between air masses. Wind Shear is the variation in wind speed and direction. It appears in the transitions between moving air masses, or between these and solid or liquid surfaces. Gusts are associated with atmospheric turbulence.

\subsection{Motivation}

The study we present is part of a wider effort to extend aircraft and, in particular, Unmanned Aerial Vehicle (UAV) endurance.

Currently, UAVs are a key element in modern military. There is also a perception that the potential for UAV civil activities is enormous. A key difference between UAVs and manned aircraft is that their endurance is not limited by operator endurance. This allows us to take a completely different approach to UAVs' endurance problems when compared with manned aircraft.

The endurance extension may be achieved through several methods. The standard method is to increase the aircraft size and wing Aspect Ratio (AR). This leads to an increase in airframe aerodynamic efficiency and fuel capacity. Good examples are large UAVs, such as the Heron TP and the Global Hawk, which have endurances greater than 36 hours. Another possibility is to install solar power panels in the aircraft. The collected energy can then be used to power the aircraft systems and propulsion, increasing its endurance.

We envision a system that is able to harvest energy from the airflow, without the need to redesign the aircraft. To produce such a result we need, for example, to evaluate the potential value of such a method, define how the aircraft should fly to harness the airflow energy, and know how to estimate the airflow parameters of interest. But before we study all those problems, we are first required to know the airflow, i.e., to have models of the flow field phenomena.

This study is a survey on atmospheric flow field phenomena models, establishing the basis for the development of all the tools necessary to harvest energy from the airflow. The models discussed are not the large time-space 
scale phenomena, widely studied by the atmospheric sciences community. The phenomena we discuss are the small set for which the characteristics match the UAVs capabilities and requirements. The models should present a low computational load, to allow a timely handling by an onboard computer. Further, the current operation areas of an UAV are usually on the order of miles or tens of miles, narrowing the choice to small space scale phenomena. Another issue is the quick aircraft dynamics that may allow airflow energy harvesting, when compared with most atmospheric phenomena time scales. This requires the aircraft to maneuver in a small area, narrowing the compatible phenomena. As such, we focus on the three types of airflow phenomena that show most potential: thermals, wind shear and gusts.

\subsection{Literature Review}

Thermals are updrafts (rising air masses) created by temperature variations (fig. 1). Wind shear is an air layer which presents a flow gradient (fig. 2). It appears between fluid masses moving with different speeds or in different directions. Gusts are transitory phenomena associated with the atmospheric turbulence.

For thermals, there are simple models based on a Gaussian curve [1, 2] and more detailed models describing Chimney Thermals [3] and Bubble Thermals [4]. None of these models predict the effects of the interaction of horizontal wind with the updraft flow field. Some are also unrealistic, in the sense that they do not respect air mass conservation, predicting that more air ascends than what descends. The studied atmospheric phenomena models are derived mainly from bird flight observations, glider pilot's observations and fluid dynamics theory.

There are several models for wind shear phenomena. The most widespread is the Surface Wind Shear model [5,6]. This model is mostly used to analyze the effect of the wind gradient near the ground during takeoff and landing operations. There are also general models $[7,8]$ for a constant wind gradient and quadratic models [9] that may represent either the constant wind gradient or wind shear transitions with varying wind gradient. There is no model to describe consistently the whole Layer Wind Shear with its transitions near the upper and lower air masses, and the core constant gradient. Also, there is no model to predict the wind gradient generated at mountain ridges.

In the atmospheric modeling community, there is wealth of knowledge primarily about large scale phenomena, both in space and time. Nevertheless, we were unable to find a comprehensive survey of the particular flow field 


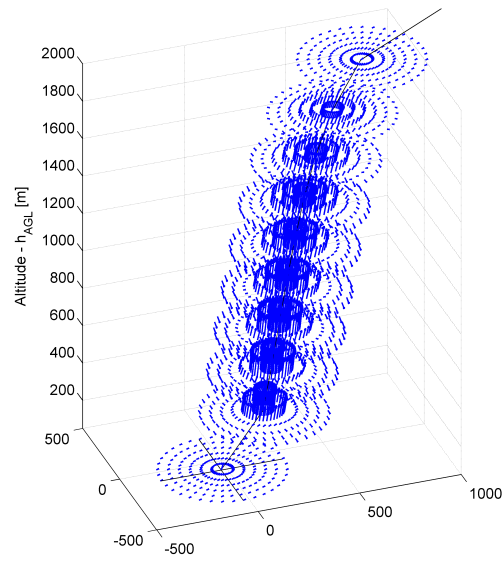

Figure 1: Column thermal updraft 3D representation.

phenomena that may be useful for flight energy harvesting. In sections 3, 4 , and 5 we discuss in more detail current shear wind, thermal updraft, and gust models, with their advantages and handicaps.

\subsection{Original Contributions}

The original contributions of this work are as follows.

Atmospheric Flow Field Models Survey This work studies and compares atmospheric flow field phenomena models useful to the analysis of flight energy harvesting. We describe several models which represent thermal updrafts, wind shear, or gust phenomena.

Improved Chimney Thermal Model This work improves the Chimney Thermal model. We include modeling features for the effect of the wind on the thermal column. This model enables the prediction of the updraft field and its dynamics, with a leaning and/or drifting Chimney Thermal.

New Bubble Thermal Model We present a new model for Bubble Thermals. This model enables the analysis of a Bubble Thermal flow field, which respects air mass conservation. Similar to the Chimney Thermal model, the new Bubble Thermal model accounts for the wind over the Bubble Thermal flow field. Furthermore, the mathematical structure of the model facilitates the computation of flow field predictions, reducing its computational load. 


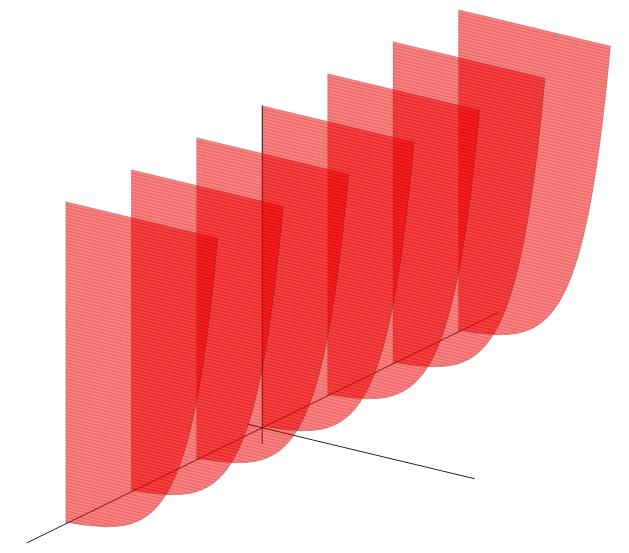

Figure 2: Surface Wind Shear 3D representation.

New Layer Wind Shear Model We present a new wind shear model for interacting air mass layers. This model enables the prediction of several wind gradients, depending on the relative position between the point of interest and the Layer Wind Shear limits. The computational characteristics of the model are simple, facilitating analysis.

New Ridge Wind Shear Model This work also develops a new wind shear model for the interaction between a flowing air mass and a mountain ridge. The Ridge Wind Shear model predicts the wind gradient along the vertical axis, from the ridge surface level to higher altitudes, and over the horizontal axis along the wind direction, to the leeward side of the ridge. This model allows the analysis of the flow field around mountain ridges.

Throughout this survey the compared characteristics of the different models described define:

1. if they are easier to handle mathematically, and probably more suitable for preliminary analysis or faster computation;

2. if they present higher fidelity, which is important for realistic simulations;

3. or if they represent different phenomena altogether. 
We describe several thermal updraft models with different complexity and realism levels. We compare the thermal updraft models over several characteristics, such as the updraft flow field shape and the relation between the thermal diameter and the updraft speed. The discussed model characteristics are important to select adequate models for the analysis purpose and constrains. As an example, if the analysis is only regarding the thermal updraft field over a constant altitude horizontal plane, the full 3D structure of the models may not be important, as well as the with influence on the vertical shape of the flow field.

We also present and compare several wind shear models. Some of these models are more suitable for the analysis of general wind shear, others for wind shear affected by the terrain, and other for Layer Wind Shear. Similar to the thermal models, these models' characteristics play a key role in their selection to a certain analysis. As an example, one may prefer to use a general wind shear model to characterize a short vertical range, due to its simplicity, or a more comprehensive model to capture the full vertical range gradient.

\subsection{Paper structure}

The remainder of this paper is as follows. We define a common nomenclature for the flow field phenomena models in Section 2. Section 3 lays out the wind shear models and compares them. We describe the thermal updraft model in Section 4, where we also compare the models structures and features. In Section 5 we discuss several gust models, with their advantages and disadvantages. We close with the conclusions (Sec. 6) and an overview of envisioned future work (Sec. 7).

\subsection{Acronyms}

AR Aspect Ratio

pdf probability density function

UAV Unmanned Aerial Vehicle 


\section{Atmospheric Phenomena}

Definition 1. A flow field is defined at a given position $\mathbf{x}=[x, y, z]^{\top}$ and time $t$ by the air flow velocity vector $\mathbf{w}$ and gradient matrix $\mathbf{J}_{W}$ :

$$
\begin{aligned}
\mathbf{w}:= & {\left[\begin{array}{l}
W_{x}(\mathbf{x}, t) \\
W_{y}(\mathbf{x}, t) \\
W_{z}(\mathbf{x}, t)
\end{array}\right] } \\
\mathbf{J}_{W}:= & {\left[\begin{array}{lll}
\frac{\partial W_{x}(\mathbf{x}, t)}{\partial x} & \frac{\partial W_{x}(\mathbf{x}, t)}{\partial y} & \frac{\partial W_{x}(\mathbf{x}, t)}{\partial z} \\
\frac{\partial W_{y}(\mathbf{x}, t)}{\partial x} & \frac{\partial W_{y}(\mathbf{x}, t)}{\partial y} & \frac{\partial W_{y}(\mathbf{x}, t)}{\partial z} \\
\frac{\partial W_{z}(\mathbf{x}, t)}{\partial x} & \frac{\partial W_{z}(\mathbf{x}, t)}{\partial y} & \frac{\partial W_{z}(\mathbf{x}, t)}{\partial z}
\end{array}\right] . }
\end{aligned}
$$

In this work the flow field variables are written in the ground reference frame, meaning that $W_{x}(\mathbf{x}, t)$ is the flow velocity component towards the North, $W_{y}(\mathbf{x}, t)$ is the flow velocity component towards the East, $W_{z}(\mathbf{x}, t)$ is the downward flow velocity component.

Definition 2. The wind vector is the horizontal component of the air velocity vector at a given position and time:

$$
\mathbf{w}_{H}:=\left[\begin{array}{l}
W_{x}(\mathbf{x}, t) \\
W_{y}(\mathbf{x}, t)
\end{array}\right] .
$$

Further, we will refer to the flow field vertical velocity as updraft or downdraft, for a negative or positive vertical air flow, respectively.

Definition 3. An updraft is the flow field vertical velocity at a given position and time:

$$
\mathbf{w}_{U p d}:=-\mathbf{w}_{z}=-W_{z}(\mathbf{x}, t)
$$

\section{Wind Shear}

Wind shear is the atmospheric phenomenon which occurs on thin layers separating two regions where the predominant air flow is different. This difference can be either in speed, in direction, or in both speed and direction. The air layer between these regions usually presents a smooth and consistent gradient in the flow field. In general it is defined by a gradient matrix $\mathbf{J}_{W}$ (1b) with any non-zero element:

$$
\frac{\partial W_{a}(\mathbf{x}, t)}{\partial b} \neq 0, a, b=x, y, z
$$


This gradient may be exploited by UAVs $[4,2,10]$ as it is by birds $[5,11,12]$.

Generally, the wind shear phenomena can be classified by directionality as horizontal or vertical shear. Horizontal wind shear is a variation of the air flow with altitude. It exists near a surface (ground or water) [5, 11, 12], over inversion layers, on the limits of the jet stream [7] and over geographic obstacles [10]. Vertical wind shear is a variation of the air flow with the horizontal position $[x, y]$. It appears across weather fronts, near the coast, and in the vicinity of mountain ranges.

We focus on horizontal wind shear, as surface, inversion, and jet stream shear are quite steady phenomena $[13,5,8]$, i.e., their time scale is on the order of hours, days or months, resulting in a relevant energy harvest potential for soaring aircraft. As stated before, the horizontal wind shear is the variation of the wind with altitude:

$$
\frac{\partial W_{a}(\mathbf{x}, t)}{\partial z} \neq 0, a=x, y .
$$

In horizontal wind shear we distinguish Surface, Layer and Ridge Wind Shear, as the flow gradient is different for each phenomenon. The first two phenomena take place over large areas, which makes them difficult to characterize as a whole. For that reason our approach is to simplify the phenomena to uniaxial $(z)$ wind vector variations. The Ridge Wind Shear depends greatly on the distance to the ridge crest, and so its model is defined over the plane perpendicular to the ridge.

For the sake of simplicity, and because in this section we regard only the horizontal flow, we will refer to the wind vector as $\mathbf{w}$ and to its total speed as $W$.

\subsection{Surface Wind Shear}

Surface Wind Shear is a special case of horizontal shear where instead of two air mass regions we have one air mass region and a surface. The surface is usually still or moving at very low speeds relatively to the general air mass, as is the case of water surfaces. Surface Wind Shear enables albatross to fly thousands of kilometers over the ocean almost without flapping their wings $[11,12]$. Surface Wind Shear is also known in the aviation community mainly by its effects on aircraft landing and take-off operations. The reduction of flow speed towards the ground causes the aircraft airspeed to decrease in the same amount, if no compensation is applied. This effect can induce stall, 


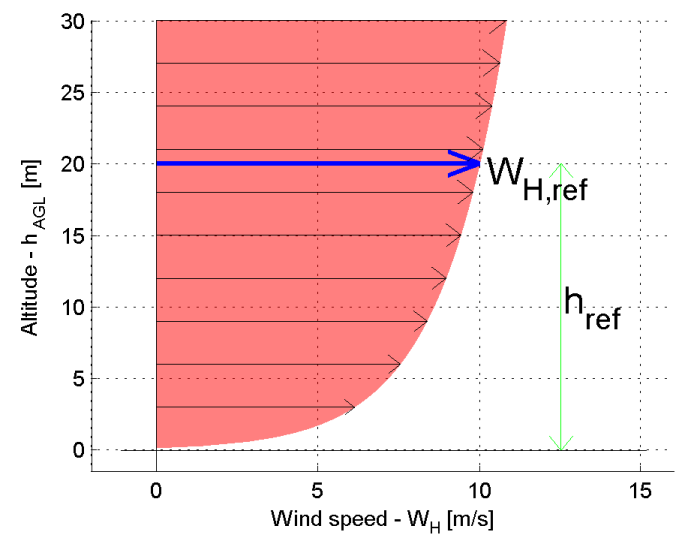

Figure 3: Surface Wind Shear profile.

leading to possibly catastrophic results. The wind shear layer starts at the ground level and may be modeled by $[5,6]$ :

$$
W=W_{h_{r e f}} \frac{\ln \left(h / h_{0}\right)}{\ln \left(h_{r e f} / h_{0}\right)},
$$

where $W$ is the total wind speed and $W_{h_{r e f}}, h_{r e f}$, and $h_{0}$ are reference values. $W_{h_{\text {ref }}}$ is the reference wind speed at a reference altitude $h_{r e f}$ away from the surface. $h_{0}$ defines the shape of the flow gradient, reflecting the surface properties, like irregularity, roughness and drag. In the Military Specification MIL-F-8785C [6]:

$$
\begin{aligned}
& h_{\text {ref }}=6 \mathrm{~m} \\
& h_{0}= \begin{cases}0.15 & \text { for Category } C \text { flight phases }, \\
2.0 & \text { otherwise }\end{cases}
\end{aligned}
$$

if $1 m \leq h \leq 300 m$. Category $\mathrm{C}$ flight phases are the terminal flight phases, which include takeoff, approach, and landing, as defined in reference [6].

\subsection{Layer Wind Shear}

Layer Wind Shear is the most general wind shear type. It can represent both a horizontal or a vertical wind shear, although in this work we focus on the horizontal shear. Two of the most common atmospheric phenomena associated with Layer Wind Shear are the Inversion Layer and the Jet 
Stream. An Inversion Layer is characterized by, as the name indicates, an inversion of the temperature gradient with altitude. Often the Convective or Mixed-Layer, the lowest in the atmosphere, is separated from the upper Troposphere layers by an Inversion Layer. If the Inversion Layer is thin enough and the flow of the separated air masses is different enough, the generated gradient may be strong enough to provide aircraft with the energy necessary to maintain flight. The Jet Stream phenomenon is characterized by a region of high-speed winds. The regions between the Jet Stream core and slower wind currents exhibit a wind gradient [8]. Glider pilots observe Layer Wind Shear sometimes above 3 knots per 1000 feet [13].

Sachs and da Costa [8] defined a model for the Layer Wind Shear observed below Jet Stream regions. The model presents a constant wind gradient, with the wind speed converging to the Jet Stream speed:

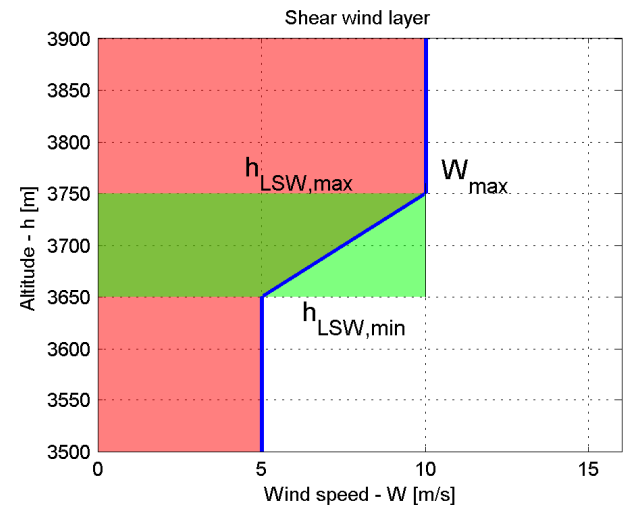

(a) Wind speed profile

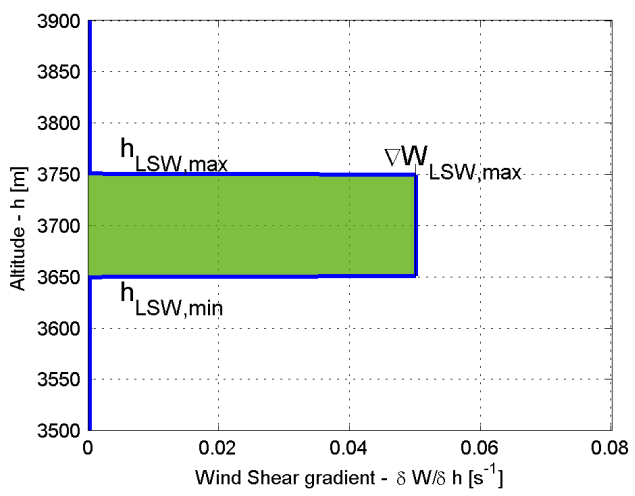

(b) Wind speed gradient

Figure 4: Sach-daCosta Layer Wind Shear model.

$$
W(h)=W_{\text {JetStream }}+\frac{\Delta W_{L W S}}{\Delta h}\left(h-h_{\text {JetStream }}\right),
$$

where $W_{\text {JetStream }}$ is the Jet Stream speed, $h_{\text {JetStream }}$ is the lower boundary of the Jet Stream core, and $\frac{\Delta W_{L W S}}{\Delta h}$ is the wind shear vertical gradient. This model does not represent the wind gradient over the transition regions, i.e., the regions where the wind speed stabilizes (fig. 7).

We now introduce a new model for the Layer Wind Shear, which includes the representation of the gradient transition regions. We approximate the 
wind gradient with a Gaussian. This model shows the expected convergence to the boundary wind speeds both over the lower and upper layer limits. The model wind speed profile is illustrated in figure 5a and defined by:

$$
\mathbf{w}(h)=\mathbf{w}\left(h_{\text {min }}\right)+\frac{\Delta \mathbf{w}_{L W S}}{2}\left[1+\operatorname{erf}\left(4 \frac{h-\bar{h}_{L W S}}{\Delta h_{L W S}}\right)\right], h \in\left[h_{\text {min }}, h_{\text {max }}\right],
$$

where $h_{\min }$ and $h_{\max }$ are the wind shear layer limit altitudes, w $(h)$ is the wind vector due to the wind shear phenomenon at an altitude $h$, and:

$$
\begin{aligned}
\Delta \mathbf{w}_{L W S} & =\mathbf{w}\left(h_{\max }\right)-\mathbf{w}\left(h_{\min }\right) \\
\bar{h}_{L W S} & =\frac{h_{\max }+h_{\min }}{2} \\
\Delta h_{L W S} & =h_{\max }-h_{\min } .
\end{aligned}
$$

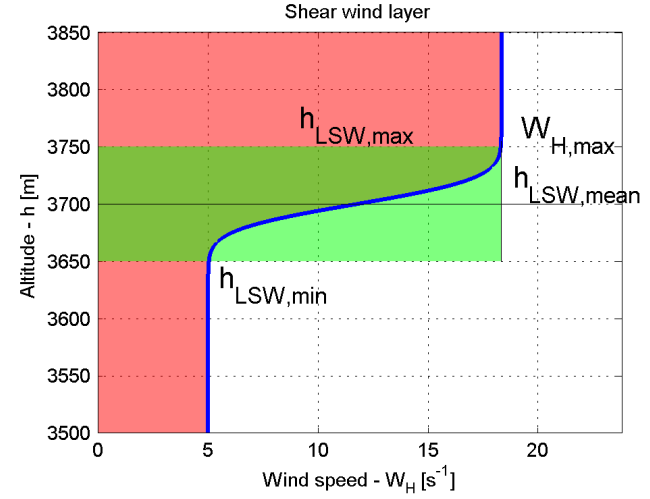

(a) Wind speed profile

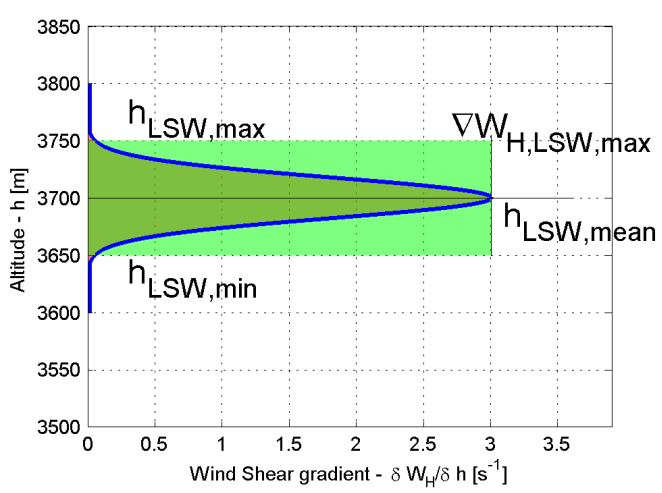

(b) Wind speed gradient

Figure 5: Gaussian Layer Wind Shear model.

The wind gradient is illustrated in figure 5b and modeled by:

$$
\left.\frac{\delta \mathbf{w}_{L W S}}{\delta h}\right|_{h}=\frac{4\left\|\Delta \mathbf{w}_{L W S}\right\|}{\Delta h_{L W S} \sqrt{\pi}} e^{-\left(4 \frac{h-\bar{h}_{L W S}}{\Delta h_{L W S}}\right)^{2}} .
$$

The erf() function 9 complicates the computation the Gaussian Layer Wind Shear model. To overcome that issue we created an alternative model, 
with simpler computational characteristics. This new model for the Layer Wind Shear is an approximation by a quadratic function:

$$
\begin{aligned}
& \mathbf{w}(h)= \begin{cases}\mathbf{w}\left(h_{\min }\right) & h \leq h_{\text {min }} \\
\mathbf{w}\left(h_{\min }\right)+2 \Delta \mathbf{w}_{L W S}\left(\frac{h-h_{\min }}{\Delta h_{L W S}}\right)^{2} & h \in\left(h_{\min }, \bar{h}_{L W S}\right] \\
\mathbf{w}\left(h_{\max }\right)-2 \Delta \mathbf{w}_{L W S}\left(\frac{h_{\max }-h}{\Delta h_{L W S}}\right)^{2} & h \in\left(\bar{h}_{L W S}, h_{\min }\right) \\
\mathbf{w}\left(h_{\max }\right) & h \geq h_{\max } .\end{cases} \\
&\left.\frac{\delta \mathbf{w}_{L W S}}{\delta h}\right|_{h}= \begin{cases}0 & h \leq h_{\min } \\
4 \Delta \mathbf{w}_{L W S} \frac{h-h_{\min }}{\Delta h_{L W S}^{2}} & h \in\left(h_{\min }, \bar{h}_{L W S}\right] \\
4 \Delta \mathbf{w}_{L W S} \frac{h_{\max }-h}{\Delta h_{L W S}^{2}} & h \in\left(\bar{h}_{L W S}, h_{\min }\right) \\
0 & h \geq h_{\max } .\end{cases}
\end{aligned}
$$

As the Gaussian model, the quadratic model converges to the boundary wind speeds at the limits (fig. 6a). Its advantages over the Gaussian model are the easier computation and smooth transitions at the layer limits.

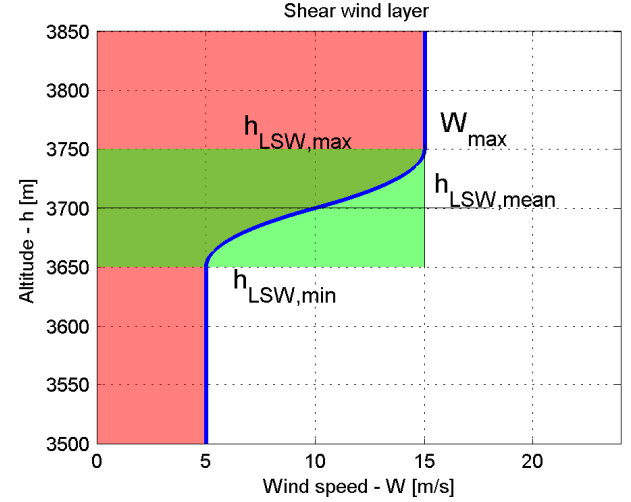

(a) Wind speed profile

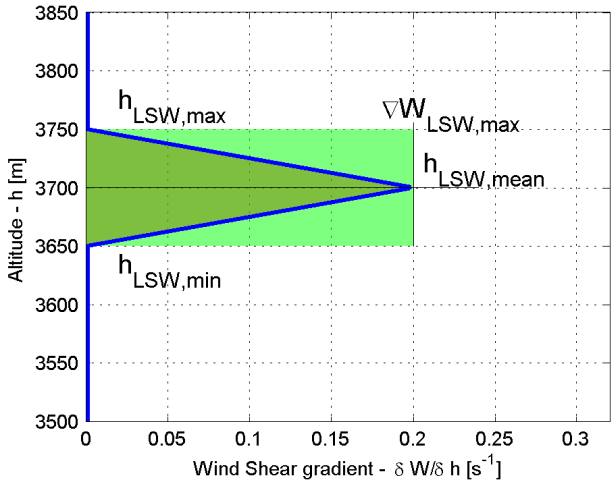

(b) Wind speed gradient

Figure 6: Quadratic Layer Wind Shear model.

Both the Gaussian and the quadratic models for the Layer Wind Shear include regions representing a gradient transition in the bottom and the top of the wind shear, and an almost constant gradient region at the center of the wind shear. Sach and da Costa [8] report the existence of large regions with a constant vertical gradient. We now extend the quadratic Layer Wind 
Shear model to include a linear part. This linear section of the wind shear represents a variable sized region with constant vertical gradient. Further, this model allows a definition of gradient transition regions with different sizes (fig. 7). The wind velocity and gradient field of this linear-quadratic

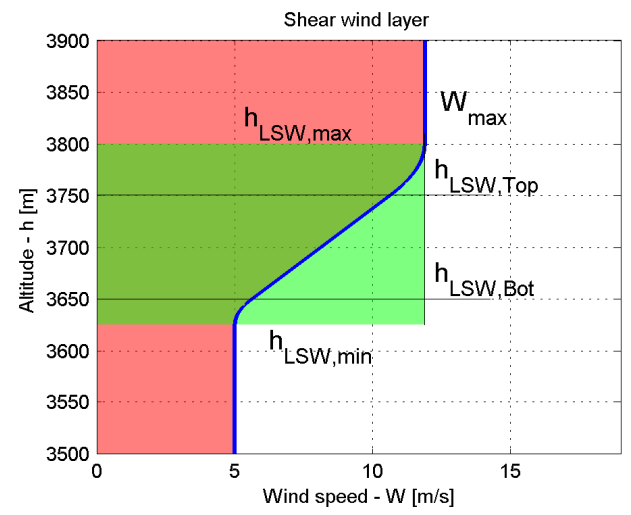

(a) Wind speed profile

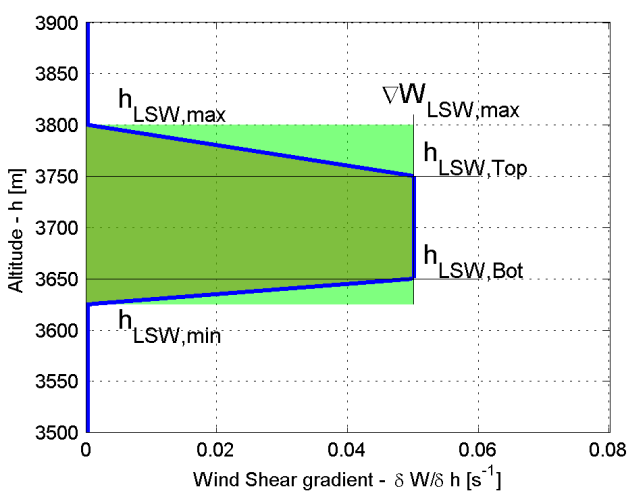

(b) Wind speed gradient

Figure 7: Linear and quadratic Layer Wind Shear model.

Layer Wind Shear model is defined as follows:

$$
\begin{aligned}
& \mathbf{w}(h)= \begin{cases}\mathbf{w}\left(h_{\min }\right) & h \leq h_{\min } \\
\mathbf{w}\left(h_{\min }\right)+\left(\frac{\delta \mathbf{w}}{\delta h}\right)_{\max } \frac{\left(h-h_{\min }\right)^{2}}{2 \Delta h_{L W S, B o t}} & h \in\left(h_{\min }, h_{L W S, B o t}\right) \\
\mathbf{w}\left(h_{L W S, B o t}\right)+\left(\frac{\delta \mathbf{w}}{\delta h}\right)_{\max }\left(h-h_{L W S, B o t}\right) & h \in\left[h_{L W S, B o t}, h_{L W S, T o p}\right] \\
\mathbf{w}\left(h_{\max }\right)-\left(\frac{\delta \mathbf{w}}{\delta h}\right)_{\max } \frac{\left(h_{\max }-h\right)^{2}}{2 \Delta h_{L W S, T o p}} & h \in\left(h_{L W S, T o p}, h_{\min }\right) \\
\mathbf{w}\left(h_{\max }\right) & h \geq h_{\max } .\end{cases} \\
&\left.\frac{\delta \mathbf{w}_{L W S}}{\delta h}\right|_{h}= \begin{cases}0 & h \leq h_{\min } \\
\left(\frac{\delta \mathbf{w}}{\delta h}\right)_{\max } \frac{h-h_{\min }}{\Delta h_{L W S, B o t}} & h \in\left(h_{\min }, h_{L W S, B o t}\right) \\
\left(\frac{\delta \mathbf{w}}{\delta h}\right)_{\max } & h \in\left[h_{L W S, B o t}, h_{L W S, T o p}\right] \\
\left(\frac{\delta \mathbf{w}}{\delta h}\right)_{\max } \frac{h_{\max }-h}{\Delta h_{L W S, T o p}} & h \in\left(h_{L W S, T o p}, h_{\min }\right) \\
0 & h \geq h_{\max }\end{cases}
\end{aligned}
$$

where $\left(\frac{\delta \mathbf{w}}{\delta h}\right)_{\max }$ is the maximum vertical gradient of the wind shear, $h_{L W S, B o t}$ is the maximum altitude of the bottom gradient transition region, $h_{L W S, T o p}$ 
is the minimum altitude of the top gradient transition region, $\Delta h_{L W S, B o t}$ is the thickness of the bottom gradient transition region, $\Delta h_{L W S, T o p}$ is the thickness of the top gradient transition region, and $\mathbf{w}\left(h_{L W S, B o t}\right)=\mathbf{w}\left(h_{\min }\right)+$ $\left(\frac{\delta \mathbf{w}}{\delta h}\right)_{\max } \Delta h_{L W S, B o t} / 2$.

\subsection{Ridge Wind Shear}

The Ridge Wind Shear is a special case of the Layer Wind Shear, that appears on the leeward side of a mountain. It is generated when the free moving air finds a large obstacle (the ridge) and, while flowing over it, is not capable of accelerating instantaneously the air mass behind the ridge. This wind shear type is the most used by radio controlled gliders, as it is strong enough, maintains a constant position and the trajectory required to use it is safe enough to avoid ground collisions [10]. From Parle's wind velocity measurements [10] it is clear that the vertical gradient appears over the leeward side of the ridge.

We found no model in the literature representing the Ridge Wind Shear phenomenon. As such, we developed a new model based on Parle's wind velocity measurements. The hypothetical model which approximately fits Parle's data is defined as:

$$
\begin{aligned}
\mathbf{w}_{R W S}=\mathbf{w}_{\infty} e^{-\frac{\lambda x}{W_{\infty}}}+\left(1-e^{-\frac{\lambda x}{W_{\infty}}}\right) \cdot \mathbf{w}_{L W S}(h) \mid & h_{\min }=h_{\text {Ridge }} \\
& h_{\max }=h_{\text {Ridge }}+k_{1} x \\
& \mathbf{w}\left(h_{\min }\right)=k_{2} \mathbf{w}_{\infty} \\
& \mathbf{w}\left(h_{\max }\right)=\mathbf{w}_{\infty}
\end{aligned}
$$

where $\mathbf{w}_{L W S}(h)$ is the Layer Wind Shear gradient model. In this model the gradient strength varies as a first order response with the spatial coefficient $\lambda$. The gradient thickness is modeled as proportional $\left(k_{1}\right)$ to the distance from the ridge crest. The gradient bottom wind speed is proportional $\left(k_{2}\right)$ to the undisturbed wind speed $\left(\mathbf{w}_{\infty}\right)$. Note that this model has 3 degrees-offreedom: $\lambda, k_{1}$, and $k_{2}$.

We now consider $\lambda, k_{1}$, and $k_{2}$ relationship with other physical variables. Given that we only have access to Parle's wind velocity measurements, we can only conjecture about this relationship. Our hypothetical interpretation is as follows. $k_{1}$ is similar to the terrain slope on the windward side of the ridge. Similarly, $k_{2}$ is related with the terrain slope on the ridge leeward side. To the best of our knowledge, $\lambda$ should depend on the ridge abruptness. This means that a sharp ridge results in a larger $\lambda$. 


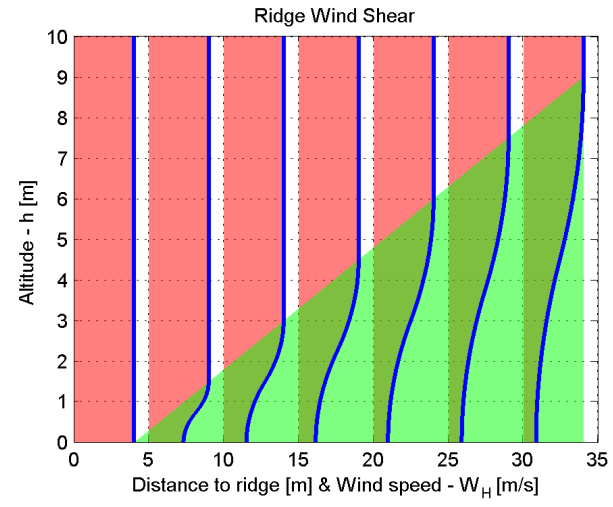

(a) Wind speed profile

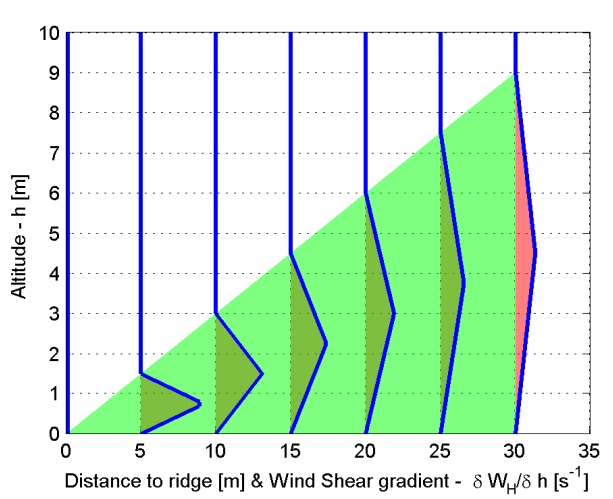

(b) Wind speed gradient

Figure 8: Variation of wind speed over a Ridge Wind Shear with altitude and distance to the ridge crest.

Figure 8 illustrates the wind speed variation within the Ridge Wind Shear. Notice the decrement of the minimum wind speed with the distance to the ridge crest, modeled by the first order response. It is also clear that the wind gradient is stronger closer to the ridge, indicating that this is the best area to harvest energy, as experienced by radio controlled glider pilots.

\subsection{Generic Wind Shear}

Zhao [9] proposed a wind shear model that is able to represent a linear gradient, as well as an exponential-like gradient or a logarithmic-like gradient. This model defines a quadratic wind speed profile with an average slope $\left(\frac{\Delta W}{\Delta h}\right)$ over an altitude range $\left(\left[0, h_{\max }\right]\right)$ :

$$
W=\frac{\Delta W}{\Delta h}\left[\Upsilon \cdot h+\frac{1-\Upsilon}{\Delta h} \cdot h^{2}\right]
$$

where $\Delta h=h_{\max }-h_{\min }$ and $\Upsilon$ defines the profile shape. $\Upsilon$ is required to remain within $[0,2]$, to keep the wind profile variation within $[0, \Delta W] . \Upsilon=1$ results in a constant vertical gradient. $0 \leq \Upsilon<1$ yields an exponential-like wind profile. And $1<\Upsilon \leq 2$ yields a logarithmic-like wind profile.

This model may be extended to a $2 \mathrm{D}$ wind vector profile with an average variation of $\frac{\Delta \mathbf{w}}{\Delta h}$ over an altitude range $\left(\left[h_{\min }, h_{\max }\right]\right)$ :

$$
\mathbf{w}=\mathbf{w}\left(h_{\text {min }}\right)+\frac{\Delta \mathbf{w}}{\Delta h}\left[\Upsilon\left(h-h_{\text {min }}\right)+\frac{1-\Upsilon}{\Delta h}\left(h-h_{\text {min }}\right)^{2}\right],
$$




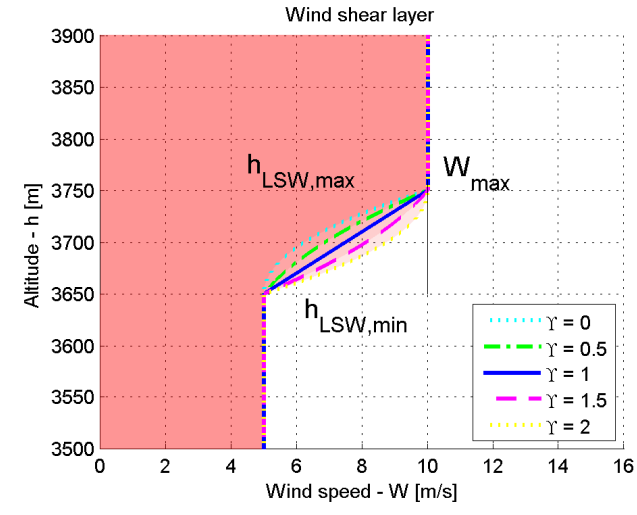

(a) Wind speed profile

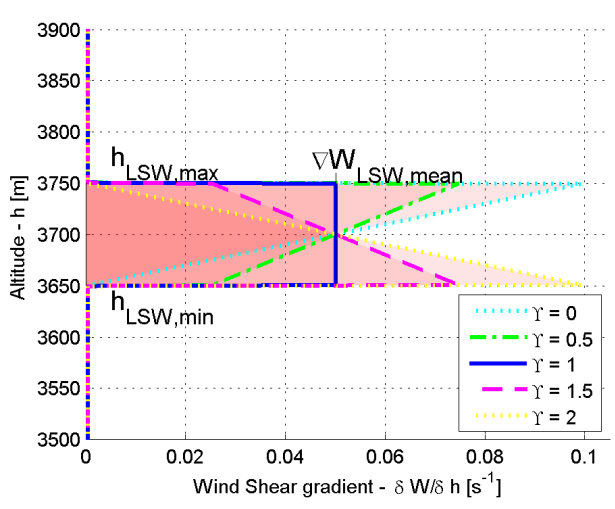

(b) Wind speed gradient

Figure 9: Zhao Generic Wind Shear quadratic model.

This model may approximate a Surface Wind Shear with $1<\Upsilon \leq 2$. It may also approximate the linear region of a Layer Wind Shear with $\Upsilon=1$ and the transition regions with $0 \leq \Upsilon<1$.

\subsubsection{Wind Shear Models Comparison}

We now summarize the main similarities and differences among the presented wind shear models. The models diverge in the vertical gradient variation, the bounding altitudes and the definition of a horizontal gradient.

Table 1: Wind Shear Models Comparison

\begin{tabular}{|c|c|c|c|c|}
\hline Model & WS type $e^{1 \star}$ & Vert. grad. ${ }^{2 \star}$ & Low. alt. bound $d^{3 \star}$ & Spatial def. ${ }^{4 \star}$ \\
\hline Surface WS [6] & SWS $^{5 \star}$ & Logarithmic & Surface & $1 \mathrm{D}$ \\
\hline Sachs-daCosta LWS ${ }^{9 \star}$ & $\mathrm{LWS}^{6 \star}$ & Linear & Air flow layer & 1D \\
\hline Bencatel G. LWS ${ }^{10 \star}$ & $\mathrm{LWS}^{6 \star}$ & Gaussian & Air flow layer & 1D \\
\hline Bencatel LQ. LWS ${ }^{11 \star}$ & $\mathrm{LWS}^{6 \star}$ & Lin. \& Quad. & Air flow layer & $1 \mathrm{D}$ \\
\hline Bencatel RWS ${ }^{12 \star}$ & RWS $^{7 \star}$ & Quadratic & Ridge & $2 \mathrm{D}$ \\
\hline Zhao WS $^{13 \star}$ & $\mathrm{S} / \mathrm{LWS}^{8 *}$ & Quadratic & Any $^{14 \star}$ & 1D \\
\hline
\end{tabular}

$1 \star$ Type of wind shear represented.

$2 \star$ Function type of the vertical gradient.

$3 \star$ Lower altitude bound of the wind shear phenomenon.

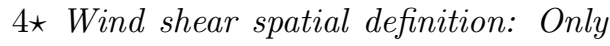
over the vertical axis (1D) or with a defined vertical and horizontal gradient (2D).

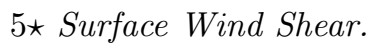




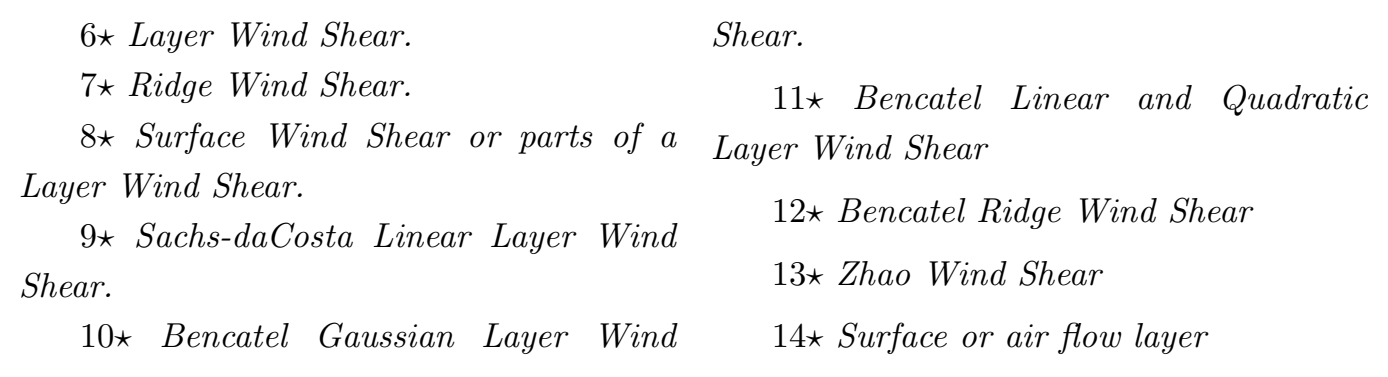

Computational complexity - This property is very important for the computational load of each model. Most of the presented models result in low computation power requirements, due to the simplicity of the functions which define them. The two models which present the greatest computational load are the Ridge Wind Shear model and the Gaussian Layer Wind Shear. The first is more complex because it represents a 2D flow field, requiring the evaluation of more variables. The Gaussian Layer Wind Shear complexity arises from the inclusion of the erf () function.

Vertical gradient variation - The Sachs-daCosta model [8] is the simplest model, but it can only represent linear gradients. This may be useful if we are analyzing just the linear part of a Layer Wind Shear or if we focus on small altitude variations, where the gradient may be considered piece-wise linear. Zhao's model [9] is almost as simple as Sachs-daCosta model, but is able to represent quadratic gradients. The Surface Wind Shear may be represented by this quadratic approximation. Different parts of the Layer Wind Shear may also be represented by this model, but not the whole phenomenon. Zhao's model may also be adequate when the goal is to observe a phenomenon for which there is no detailed model, or parts of it. The Surface Wind Shear model [6] represents the gradient through a logarithmic function, which is the widely accepted representation. The Ridge Wind Shear model uses the same gradient representation as the Layer Wind Shear model with a variable layer thickness parameter, dependent on the horizontal distance to the ridge. Both the Gaussian and Linear-Quadratic Layer Wind Shear models represent the whole Layer Wind Shear phenomenon, including the gradient variation at the bounding altitudes. The Linear-Quadratic Layer Wind Shear model is simpler to compute and allows the representation of regions with a linear gradient with an independent size, when compared to the global Layer Wind Shear thickness. 
Model specificity - The only general models are the linear Sachs-daCosta model [8] and Zhao's quadratic model [9]. All other models are specific either to Surface Layer or Ridge Wind Shear phenomena. Further, the Ridge Wind Shear model is the only one that defines a horizontal gradient together with the vertical gradient.

\section{Updraft}

Updrafts are upward moving air masses. These air masses can be small upward gusts, created by turbulence, and ranging from centimeters to a few meters. Updrafts can also be the result of large rising air mass bodies, ranging from 50 meters to kilometers. These originate from terrain topography or from thermal flows. The first type, called orographic updrafts, are generated when the wind hits a terrain slope, creating strong updrafts above the terrain. The thermal flows are generated by hot spots on the ground. These create a thermal gradient, heating the surrounding air. The density of the heating air decreases, forcing an upward movement. Thermal updrafts don't depend as much on topography or on wind as orographic updrafts.

Thermals are part of the convection flows that develop in the mixing-layer of the atmosphere, the lowest atmospheric layer, also called the convective layer. A thermal model may be characterized by three types of parameters: the atmosphere parameters, the internal parameters, and the terrain distribution. The atmosphere parameters characterize the regional environment characteristics. These are the variables that are slow varying and very similar in adjacent flight areas. The internal parameters are individual to each thermal. These parameters define the spatial effects of each specific thermal, and may be distinct for different adjacent thermals. The terrain distribution concerns the sparsity among thermals, which affects the rate of appearance of thermals in the aircraft flight path.

There are several types of thermals. Chimney Thermals, also designated as Column Thermals, are continuous columns of rising air that extend from the ground surface right up to the mixing-layer maximum altitude. Bubble Thermals are closed shells [14] of rising air. They are formed near the ground when the temperature differences are large enough to create buoyancy, like in a balloon. These shells then rise and depart from the ground. Cone [14] describes them as rising vortex rings, where there is a circulatory flow generated by a strong core updraft. This core updraft is fed by the buoyant air. When this air leaves the bubble core, it starts to cool down, becoming 
less buoyant. The cooled air then moves downward on the outside of the vortex ring, completing the cycle.

The main model features of a thermal are its position, the updraft field, representing how the vertical airspeed varies with the position relative to the thermal center, and its radius, defining where the updraft speed is null or almost null. The models we will present next differ on three main modeling features: the effect of altitude variation, the representation of the thermal skirt downdraft, and the interdependence among the updraft field and thermal radius.

\subsection{Thermals}

The simplest thermal model represents the updraft field as a 3D trapezoid, i.e., a conical trunk (Fig. 10). This model presents a core updraft, from the thermal center to its inner radius, and a decreasing updraft from the inner radius to the outer radius. Outside the thermal outer radius, there is no vertical flow velocity. This model is defined by:

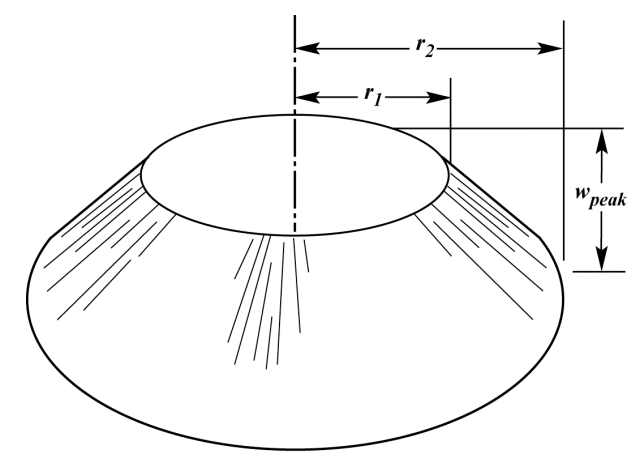

Figure 10: Updraft velocity trapezoidal model (illustration from [3])

$$
w(r)= \begin{cases}W_{z \cdot \max } & r<r_{1} \\ W_{z \cdot \max } \frac{r_{2}-r}{r_{2}-r_{1}} & r \in\left[r_{1}, r_{2}\right] \\ 0 & \text { otherwise }\end{cases}
$$

where $r$ is the distance from the thermal center, $W_{z . m a x}$ is the core updraft speed, $r_{1}$ is the thermal inner radius, and $r_{2}$ is the thermal outer radius.

The most used thermal model is based on a scaled 2D Gaussian, for its simplicity $[15,16]$. This is a simple model where the center of the thermal is 
the center of the Gaussian [1] (fig. 11):

$$
w(r)=W_{z \cdot \max } \cdot e^{-\left(r / R_{T}\right)^{2}} .
$$

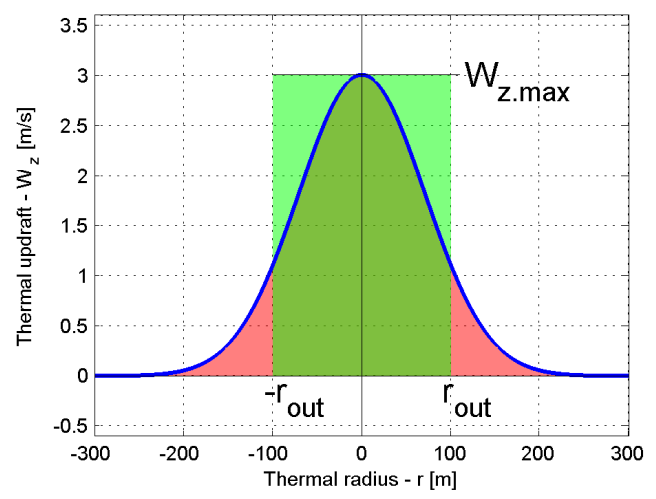

Figure 11: Thermal updraft Gaussian model

The Gaussian is scaled so that its maximum matches the maximum updraft (core updraft). Furthermore, the Gaussian variance is adjusted so that an almost null velocity is found at the thermal outer radius. Therefore, this model does not include the exterior downdraft and the thermal radius altitude dependence. However, the model is good enough to represent the thermal core at close altitudes.

Gedeon's Thermal model [2] is an extension of the Gaussian model. He adjusted the updraft function so that it presented a negative speed outside the thermal radius and a null speed at the thermal radius (fig. 12):

$$
w(r)=W_{z \cdot \max } \cdot e^{-\left(r / R_{T}\right)^{2}} \cdot\left[1-\left(r / R_{T}\right)^{2}\right] .
$$

This model only represents the thermal in the horizontal dimensions, because it does not present any dependency of the thermal diameter with the altitude. As such, it is a more realistic model of a thermal at close altitudes than the Gaussian model.

There are some references which argue that the bell-shape changes according to the size and strength of the thermal [17, 18, 19]. Observations lead to the hypothesis that there are two more prevalent thermal shape types 


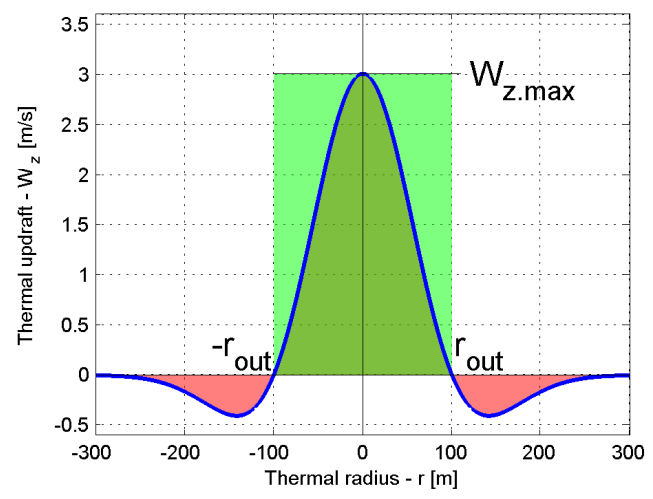

Figure 12: Thermal updraft Gedeon model

$[17,18,19]$, one with a pronounced maximum (type "b") and another with several maxima (type "a"), almost as a plateau (fig. 13). According to the same source, type " $b$ " thermals appear more frequently in lower energy environments. Type "a" is observed more frequently when the temperature gradient is larger, and seems to be the result of a merge between several type "b" thermals. Furthermore, Lenschow and Stephens [20] state that the magnitude of vertical velocity variation within a thermal may be larger than the magnitude of the overall mean updraft velocity.
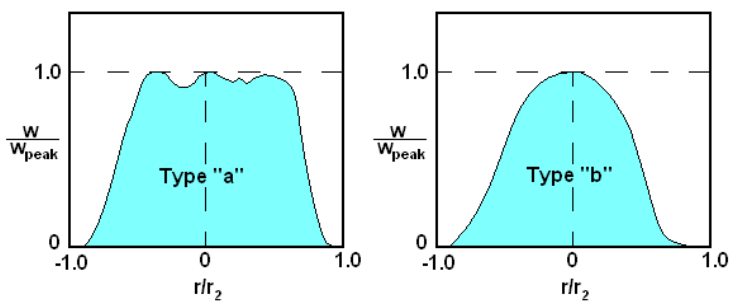

Figure 13: Updraft velocity magnitude

Lawrance and Sukkarieh [4] present a toroidal model as an hypothesis for a Bubble Thermal flow structure. It represents the vortex ring proposed by Cone [14] in all three dimensions. Further, the model represents the flow field in every direction instead of only the vertical flow.

Allen [3] presents a detailed updraft model developed at NASA Dryden Flight Research Center with real flow measurements. This model represents 


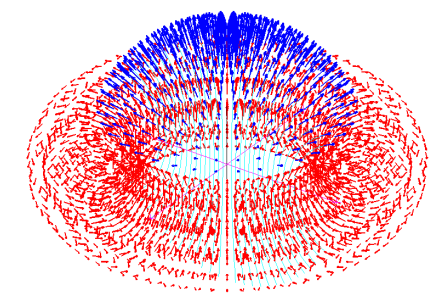

Figure 14: Bubble Thermal

a continuous Chimney Thermal in all three dimensions. It relates the atmospheric parameters with their effect on the vertical flow at different altitudes. There is no prediction on the horizontal flow velocity, but the predicted vertical flow respects the mass conservation along any horizontal plane.

Thermals will lean or drift with the prevalent direction of the wind [13]. The organization in Chimney or Bubble is affected by the existing wind shear. The fraction between the thermal strength and the wind shear plays an important role on the organization definition [13].

There are different theses about which is the most prevalent type of thermals, Chimney (fig. 15) or Bubbles (fig. 14). [13] states that the Chimney Thermal type is the most prevalent kind of thermals, indicating that Bubbles appear when the heating is slow or intermittent. This may happen due to the radiated surface properties or due to moving cloud shadows. On the other hand, Cone [14] defends that Bubble Thermals are the most frequent. This thesis is sustained by predictions of vortex theory and experiments that attested to the formation of rising vortex rings. Furthermore, continuous Chimney Thermals would require a continuous air supply near the ground, which would be sensed as a continuous ground wind. However, the appearance of thermals is not usually associated with sustained wind. A more common phenomenon is sudden wind gusts, that can be associated with the formation of a Bubble Thermal. Groups of soaring birds take off in what seems like a reaction to these sudden gusts. Another observation that sustains the prevalence of Bubble Thermals is the fact that birds usually cluster in a short altitude range when soaring. Birds below the main cluster frequently have to flap their wings to reach the soaring group. 


\subsection{Chimney Thermal - Allen Model}

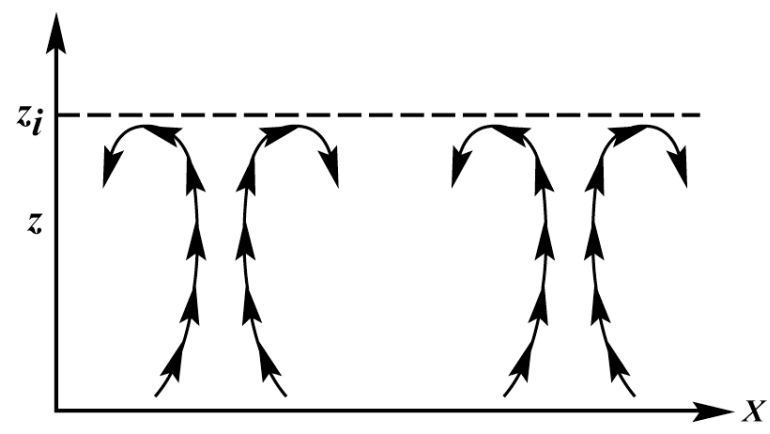

Figure 15: Chimney Thermal representation with mixing-layer thickness $\left(z_{i}\right)$ (illustration from [3])

Allen [3] developed a thermal model which represents the updraft strength in all three dimensions. His model defines the radius and the updraft field at each altitude as a function of two atmospheric parameters: the mixinglayer thickness and the convective velocity scale. The mixing-layer thickness $\left(z_{i}\right)$ is the mixing-layer maximum altitude (fig. 15), where the convection flows appear. As such, $z_{i}$ is also the maximum altitude for the thermal activity. This depends on the ground temperature $T_{S}$ and on the predawn air temperature profile [3] (fig. 16):

$$
z_{i}=f\left(T_{S}, \text { Predawn air temperature profile }\right) .
$$

The mixing-layer thickness $\left(z_{i}\right)$ varies slowly with the location, presenting a low spatial variation frequency. The thickness is usually 0 at dawn, rises to the day maximum at late afternoon [17] and returns to 0 during the night. Therefore, the regional variation time constant should be high, on the order of hours.

The convective velocity scale $\left(w^{\star}\right)$ is a reference which indicates the predicted velocity magnitudes in and around a thermal. Again reference [3] shows that this velocity is a function of the mixing-layer thickness $\left(z_{i}\right)$, the surface virtual potential temperature flux $\left(Q_{O V}\right)$, the ground temperature $T_{S}$, and the static pressure at ground level $(p)$ [3]. Furthermore, the virtual potential temperature flux is a function of the net radiation at the surface $\left(Q_{S}\right)$, the air relative humidity $(r h)$, the saturated vapor pressure $(e s)$, and the ground temperature $T_{S}$. 
Table 2: Yearly convective lift statistical properties (from reference [3])

\begin{tabular}{|c|c||c|c|c|}
\hline Description & $w^{\star}, \mathrm{m} / \mathrm{s}$ & $\mu_{z_{i}}-\sigma_{z_{i}}, \mathrm{~m}$ & $\mu_{z_{i}}, \mathrm{~m}$ & $\mu_{z_{i}}+\sigma_{z_{i}}, \mathrm{~m}$ \\
\hline \hline$\mu_{w^{\star}}-2 \sigma_{w^{\star}}$ & 0.46 & 25.6 & 53.6 & 97.4 \\
\hline$\mu_{w^{\star}}-\sigma_{w^{\star}}$ & 1.27 & 150 & 210 & 1007 \\
\hline$\mu_{w^{\star}}$ & 2.56 & 767 & 1401 & 2319 \\
\hline$\mu_{w^{\star}}+\sigma_{w^{\star}}$ & 4.08 & 2134 & 2819 & 3638 \\
\hline$\mu_{w^{\star}}+2 \sigma_{w^{\star}}$ & 5.02 & 2913 & 3647 & 4495 \\
\hline
\end{tabular}

$w^{\star}$ seems to have a spatial and temporal dependency similar to $z_{i}$. The only extra factor is the wind speed, which seems to disrupt any thermal if blowing above $12.87 \mathrm{~m} / \mathrm{s}$ (in the Mojave Desert [3]), but also favors organized thermal convection if above $5 \mathrm{~m} / \mathrm{s}$ [13].

Allen [3] describes the yearly and monthly statistics for $w^{\star}$ and $z_{i}$ in Desert Rock, Nevada. Table 2 show the yearly statistics. These statistics are important to inference processes as a prior belief, e.g., for thermals parameters estimation.

The internal parameters are: the outer radius, and the vertical flow field, with:

$$
\begin{aligned}
w_{T} & \sim w * \\
r_{2} & \sim z_{i} .
\end{aligned}
$$

The outer radius varies from thermal to thermal, and inside the thermal with the altitude above the ground. Lenschow and Stephens [20] state that the thermal radius average at a certain altitude is a direct function of the mixing-layer thickness $\left(z_{i}\right)$, by (fig. 17):

$$
r_{2}=\max \left[10,0.2513\left(\frac{z}{z_{i}}\right)^{\frac{1}{3}}\left(1-0.25 \frac{z}{z_{i}}\right) \cdot z_{i}\right] \text {. }
$$

Note that multiplier 0.2513 is different from the one used by Allen [3], as this author corrected the calculations later on.

The equation governing the vertical velocity is [3]:

$$
w=w_{\text {peak }}\left(\frac{1}{1+\left|k_{1} \frac{r}{r_{2}}+k_{3}\right|^{k_{2}}}+k_{4} \frac{r}{r_{2}}+w_{D}\right)\left(1-\frac{w_{e}}{w_{\text {peak }}}\right)+w_{e} .
$$


Table 3: Shape constants for bell-shaped vertical velocity distribution [3]

\begin{tabular}{|c||c|c|c|c|}
\hline$\frac{r_{1}}{r_{2}}$ & $k_{1}$ & $k_{2}$ & $k_{3}$ & $k_{4}$ \\
\hline \hline 0.14 & 1.5352 & 2.5826 & -0.0113 & 0.0008 \\
\hline 0.25 & 1.5265 & 3.6054 & -0.0176 & 0.0005 \\
\hline 0.36 & 1.4866 & 4.8354 & -0.0320 & 0.0001 \\
\hline 0.47 & 1.2042 & 7.7904 & 0.0848 & 0.0001 \\
\hline 0.58 & 0.8816 & 13.972 & 0.3404 & 0.0001 \\
\hline 0.69 & 0.7067 & 23.994 & 0.5689 & 0.0002 \\
\hline 0.80 & 0.6189 & 42.797 & 0.7157 & 0.0001 \\
\hline
\end{tabular}

The constants $k_{1}, k_{2}, k_{3}$, and $k_{4}$ are defined in table 3 .

The variables $w_{\text {peak }}, w_{D}, w_{e}$, and $r_{1}$ are defined next. We first define the average updraft speed function (fig. 19):

$$
\bar{w}=w^{\star}\left(\frac{z}{z_{i}}\right)^{\frac{1}{3}}\left(1-1.1 \frac{z}{z_{i}}\right) .
$$

Now, the radius for which the updraft speed is almost constant $\left(r_{1}\right)$ comes from

$$
\frac{r_{1}}{r_{2}}=\left\{\begin{array}{ll}
0.0011 r_{2}+0.14 & r_{2}<600 \\
0.8 & \text { otherwise }
\end{array} .\right.
$$

The maximum updraft speed $\left(w_{\text {peak }}\right)$ is defined as

$$
w_{\text {peak }}=\bar{w} \frac{3 r_{2}^{2}\left(r_{2}-r_{1}\right)}{r_{2}^{3}-r_{1}^{3}} .
$$

The skirt downdraft speed $\left(w_{D}\right)$ is computed by

$$
w_{D}=\left\{\begin{array}{ll}
\frac{\bar{w} 5 \pi}{12}\left(\frac{z}{z_{i}}-0.5\right) \sin \left(\frac{\pi r}{r_{2}}\right) & r \in\left(r_{1}, 2 r_{2}\right) \vee \frac{z}{z_{i}} \in(0.5,0.9) \\
0 & \text { otherwise }
\end{array} .\right.
$$

To maintain a null regional net vertical velocity, we have to define the natural sink speed $\left(w_{e}\right)$ as:

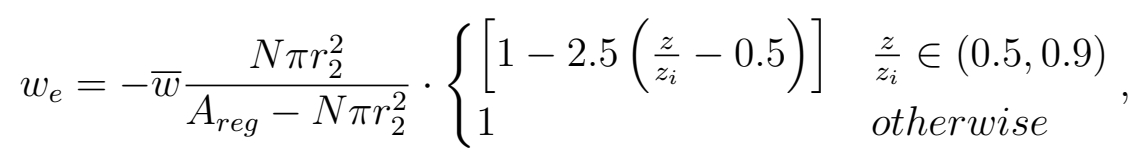

where $A_{\text {reg }}$ is the affected region. 


\subsubsection{Chimney Thermal - Interaction with the Wind}

There are three main effects the wind may have on Chimney Thermals. Wind seems to be fully disruptive for the thermals if blowing faster than 13 $\mathrm{m} / \mathrm{s}$. If it is slower, it may lean the thermal, move it, or both. The thermal drift depends both on the wind speed and on the terrain radiating properties. The drift velocity generally follows the prevailing wind, but not always [3]. If the terrain radiating properties are very uneven there is a tendency for the thermal to stay anchored to the most radiating points, usually called hot-spots, such as rocks, building roofs, asphalt, etc. When the thermal source, the lowest section of the thermal, is anchored to a hot spot or moves slower than the wind speed, the thermal has the tendency to lean to the leeward direction (fig. 20). During a thermal soaring flight thermal leaning is sometimes wrongly perceived as a drift by the whole thermal.

We now extend Allen's Chimney Thermal to account for these wind effects on the 3D flow field structure and dynamics. In this model the movement dynamics of a Chimney Thermal are captured by:

$$
\begin{aligned}
\dot{x}_{T} & =u_{T}=V_{T} \cos \psi_{T} \\
\dot{y}_{T} & =v_{T}=V_{T} \sin \psi_{T} \\
V_{T} & \sim \mathcal{N}\left(\mu_{V_{T}}, \sigma_{V_{T}}\right), \mu_{V_{T}} \in[0,\|\mathbf{w}\|] \\
\psi_{T} & \sim \mathcal{N}\left(\mu_{\psi_{T}}, \sigma_{\psi_{T}}\right),
\end{aligned}
$$

where $x_{T}$ and $y_{T}$ are the Chimney Thermal source position coordinates, $u_{T}$ and $v_{T}$ are the Chimney Thermal source drift velocities, $\mu_{V_{T}}$ and $\sigma_{V_{T}}$ are the thermal drift speed mean and standard deviation and $\mu_{\psi_{T}}$ and $\sigma_{\psi_{T}}$ are the thermal drift direction probability parameters.

The Chimney Thermal leaning may be characterized by the change in the updraft core center for different altitudes:

$$
\begin{gathered}
x_{t}(H) \approx x_{T}+\int_{0}^{H} \frac{\bar{W}_{x}(h)-u_{T}}{\bar{W}_{T, z}(h)} d h \\
y_{t}(h) \approx y_{T}+\int_{0}^{H} \frac{\bar{W}_{y}(h)-v_{T}}{\bar{W}_{T, z}(h)} d h,
\end{gathered}
$$

where $x_{t}(H)$ represents the updraft core center coordinates at an altitude above the ground $H, \bar{W}_{x}(h)$ and $\bar{W}_{y}(h)$ are the mean wind velocities away 
from the thermal at each altitude $h$, and $\bar{W}_{T, z}(h)=\bar{w}$ is the updraft mean speed at each altitude $h$. From this equation it is clear that the thermal will not lean if the source is moving at the same velocity as the wind. As stated before, and because Chimney Thermal movements depend on the terrain, the thermal will present some leaning associated with some drift.

\subsection{Bubble Thermal - Lawrance Model}

Lawrance [4] developed a Bubble Thermal model. Unlike the Chimney Thermal model developed by Allen, this model describes an unsteady upward moving phenomenon. In this model the hotter air mass rises in a bubble like structure, disconnected from the ground or the inversion layer. The model described in [4] defines a toroidal 3D flow field at a given instant (Fig. 21). The flow field model created by Lawrance is defined by:

$$
\begin{aligned}
& w_{x}=-w_{z} \frac{z}{\left(d_{H}-R\right) \cdot k^{2}} \frac{x}{d_{H}} \\
& w_{y}=-w_{z} \frac{z}{\left(d_{H}-R\right) \cdot k^{2}} \frac{y}{d_{H}} \\
& w_{z}= \begin{cases}w_{\text {core }} & x=0 \\
\frac{\cos \left(1+\frac{\pi z}{k \cdot R}\right)}{2} \frac{R \cdot w_{\text {core }}}{\pi d_{H}} \sin \left(\frac{\pi d_{H}}{R}\right) & d_{H} \in(0,2 R], \\
0 & \text { otherwise }\end{cases}
\end{aligned}
$$

where $d_{H}=\sqrt{x^{2}+y^{2}}, w_{\text {core }}$ is the bubble core updraft speed, $R$ is the distance which limits the updraft area, i.e., the area around the bubble center where the flow moves upwards, $x, y$, and $z$ are positions relative to the bubble center, and $k$ is the bubble eccentricity factor, i.e., $k=\frac{\Delta z_{\text {flow }}}{2 R}$, where $\Delta z_{\text {flow }}$ is the bubble height.

\subsubsection{Bubble Thermal - Conservative Flow Model}

The Lawrance Bubble Thermal model is not mass conservative, does not include any effects of the interaction with the prevailing wind, and does not present the possibility to represent an updraft core plateau described by some authors. Figure 22 illustrates some of the models we developed to try and overcome some of these issues. The bubble flow is only conservative if the whole bubble region can be described by streamlines, i.e., the lines "followed" by air particles in the absence of disturbances. The first two 
models are adaptations of the toroid streamline model on which the Lawrance model is also based. The first model (fig. 22a) presents sharp flow direction changes which are not realistic at all, as they would require infinite flow acceleration at those points. The second model (fig. 22b) presents more realistic streamlines. The two main handicaps are the complex computation of some of the parameters, when taking a relative position as an input, and the lack of flow near the top and the bottom of the bubble. In the next streamline model we drop the constraint for all the streamlines to be centered at the toroid center, i.e., at the updraft outer radius on the mean bubble altitude plane. Instead, we define the streamlines' center to tend to the toroid center as they approach it. The third model (fig. 22c) presents nice properties in terms of parameter computation, when taking a relative position as an input, but presents no flow near the bubble axis, when away from the central plane. The final model (fig. 22d) presents a flow tending to vertical near the bubble axis, a vortex around the updraft outer radius, and is conservative in terms of mass exchange. The computation of the streamline parameters requires an iterative process when the input to define those parameters is a relative position to the bubble center. This is shown below in equation (32).

The final model presents a flow tending to vertical near the bubble axis, as we define that the streamlines center should tend to infinite as those approach the bubble axis. We also define that the streamlines' direction should be perpendicular to a circle at a distance $R_{T}$ from the bubble center (fig. 23), where $R_{T}$ is the Bubble Thermal updraft outer radius. The streamline parameters are then defined as:

$$
\begin{aligned}
r_{\text {max }} & =\frac{R_{T}^{2}}{r_{\text {min }}} \\
r_{c t r} & =\frac{r_{\text {max }}+r_{\text {min }}}{2}=\frac{R_{T}}{\cos \xi} \\
R_{\text {stream }} & =\frac{r_{\text {max }}-r_{\text {min }}}{2}=R_{T} \tan \xi \\
d_{H} & =r_{\text {max }}-\Delta z \tan \zeta \\
\Delta z & =\left(d_{H}-r_{\text {min }}\right) \tan \zeta \\
\frac{R_{T}^{2}}{r_{\text {min }}}+r_{\text {min }} & =\frac{\Delta z^{2}+d_{H}^{2}+R_{T}^{2}}{d_{H}},
\end{aligned}
$$

where $r_{\text {min }}$ and $r_{\text {max }}$ are the minimum and maximum distances of the streamline to the Bubble Thermal axis, $r_{c t r}$ is the streamline center distance to the 
Bubble Thermal axis, $R_{\text {stream }}$ is the streamline radius, $d_{H}=\sqrt{\Delta x^{2}+\Delta y^{2}}$ is the horizontal distance of a point to the Bubble Thermal axis, $\Delta x, \Delta y$, and $\Delta z$ are the coordinates of the relative position to the Bubble Thermal center, $\xi$ is the "exit" angle of the flow at a distance $R_{T}$ from the bubble center, and $\zeta$ is the angle of a point relative to the streamline center (fig. 24). The parameter iteration need arises from $r_{\min }$ being defined by the implicit function (32f).

We may also define the streamlines such that the flow is perpendicular to an ellipse, which crosses the bubble horizontal plane at the updraft outer radius $R_{T}$. The streamline parameter may then be computed by solving:

$$
r_{\text {max }}=\frac{R_{T}^{2}\left(1-e^{2} \sin ^{2} \xi\right)}{r_{\min }} .
$$

To define the flow speed over each streamline, such that it presents a mass conservative flow, the volume rate has to be constant through each streamline:

$$
\dot{V}=c^{s t}=v r \pi d A \Leftrightarrow v_{1} r_{1}=v_{2} r_{2},
$$

where $\dot{V}$ is the volume rate, $v$ is the flow total speed, and $d A$ is an infinitesimal area on the $r-z$ plane centered at radius $r_{(.)}$. This yields a flow speed over a streamline defined by:

$$
W_{2}=W_{1} \frac{d_{H, 2}}{d_{H, 1}},
$$

where $W_{(.)}$are total flow speeds at points 1 and 2 with horizontal distances $d_{H,(.)}$ from the bubble central axis. Combining (35) with the streamline orientation at each point from (32), the flow velocity vector at each point may be defined as:

$$
\mathbf{w}=W_{z, r_{\min }, \Delta z=0} \frac{r_{\min }}{d_{H}}\left[\begin{array}{c}
\sin \zeta \frac{\Delta x}{d_{h}} \\
\sin \zeta \frac{\Delta y}{d_{h}} \\
\cos \zeta
\end{array}\right]
$$

To solve (36) and completely define the Bubble Thermal flow field one just needs to define an updraft field at the bubble mean altitude $\left(W_{z, r_{\min }, \Delta z=0}\right)$. We considered three different hypotheses. The first is based on the flow field defined by Lawrance [4], constrained to $d_{H} \in\left[0, R_{T}\right]$ and $\Delta z=0$ :

$$
W_{z, d_{H}, \Delta z=0}= \begin{cases}w_{\text {core }} & d_{H}=0 \\ \frac{w_{\text {core }}}{\pi}\left(\frac{R_{T}}{d_{H}}\right)^{\delta_{\text {Flat }}} \sin \left(\pi\left(\frac{R_{T}}{d_{H}}\right)^{\delta_{\text {Flat }}}\right) & d_{H} \in\left(0, R_{T}\right]\end{cases}
$$


where $w_{\text {core }}$ is a positive value defining the maximum updraft speed and $\delta_{\text {Flat }}$ regulates the flatness of the updraft core and the abruptness of the updraft reduction towards to outer radius, as shown on figure 25 .

The second model is based on the Gedeon updraft field [2], also constrained to $d_{H} \in\left[0, R_{T}\right]$ and $\Delta z=0$ :

$$
W_{z, d_{H}, \Delta z=0}=w_{\text {core }} e^{-\left(d_{H} / R_{T}\right)^{2 \delta_{F l a t}}} \cdot\left[1-\left(d_{H} / R_{T}\right)^{2 \delta_{\text {Flat }}}\right],
$$

where $w_{\text {core }}$ and $\delta_{\text {Flat }}$ are the same as in the first model.

The last model, Plateau Updraft, is an extension of the second, with a core constant updraft:

$$
\begin{array}{r}
W_{z, d_{H}, \Delta z=0}= \begin{cases}w_{\text {core }} & d_{H} \leq R_{\text {Plat }} \\
w_{\text {core }} e^{-\delta_{R}} \cdot\left[1-\delta_{R}\right] & d_{H} \in\left(R_{\text {Plat }}, R_{T}\right]\end{cases} \\
\text { with } \delta_{R}=\left(\frac{d_{H}-R_{\text {Plat }}}{R_{T}-R_{\text {Plat }}}\right)^{2 \delta_{\text {Flat }}},
\end{array}
$$

where $R_{\text {Plat }}$ is the core updraft plateau radius. This may be defined as in the Chimney model by equation (25):

$$
\frac{R_{\text {Plat }}}{R_{T}}=\left\{\begin{array}{ll}
0.0011 R_{T}+0.14 & R_{T}<600 \\
0.8 & \text { otherwise }
\end{array} .\right.
$$

Figure 26 illustrates all three models showing that there is almost no difference between the Lawrance and the Gedeon models. It also shows that the plateau size control on the last model gives the user an extra degreeof-freedom, possibly allowing a better match between the model and real observations.

The resulting Bubble Thermal flow field is depicted in figure 27. It is very clear that the flow closest to the bubble axis is almost vertical, while near the updraft outer radius it turns into a vortex. The continuous nature of this model is also apparent. Figure 28 illustrates the main components of the Bubble Thermal, including the core updraft and the surrounding vortex, as proposed by Cone [14].

As Bubble Thermals are usually completely detached from the ground they are more affected by the prevailing wind than the Chimney Thermals. 
The Bubble Thermals center velocity is defined as:

$$
\begin{aligned}
\dot{x}_{T} & =u_{T}=V_{T} \cos \psi_{T} \\
\dot{y}_{T} & =v_{T}=V_{T} \sin \psi_{T} \\
\dot{z}_{T} & =w_{T} \\
V_{T} & \sim \mathcal{N}\left(\mu_{V_{T}}, \sigma_{V_{T}}\right), \mu_{V_{T}}=\|\mathbf{w}\| \\
\psi_{T} & \sim \mathcal{N}\left(\mu_{\psi_{T}}, \sigma_{\psi_{T}}\right),
\end{aligned}
$$

where $x_{T}, y_{T}$, and $z_{T}$ are the Bubble Thermal center coordinates, $u_{T}, v_{T}$, and $w_{T}$ are the Bubble Thermal center drift velocities, $\mu_{V_{T}}$ and $\sigma_{V_{T}}$ are the thermal drift speed mean and standard deviation and $\mu_{\psi_{T}}$ and $\sigma_{\psi_{T}}$ are the thermal drift direction probability parameters.

If the Bubble Thermal moves with a difference velocity from the prevailing wind, its structure leans. The combined flow field from a Bubble Thermal and the prevailing wind is defined by:

$$
\mathbf{w}=W_{z, r_{m i n}, \Delta z=0} \frac{r_{\min }}{d_{H}^{\prime}}\left[\begin{array}{c}
\sin \zeta^{\prime} \frac{\Delta x^{\prime}}{d_{H}^{\prime}}+l_{x} \cos \zeta^{\prime} \\
\sin \zeta^{\prime} \frac{\Delta y^{\prime}}{d_{H}^{\prime}}+l_{y} \cos \zeta^{\prime} \\
\cos \zeta^{\prime}
\end{array}\right]+\left[\begin{array}{c}
\bar{W}_{x} \\
\bar{W}_{y} \\
0
\end{array}\right],
$$

where

$$
\begin{aligned}
& l_{x}=\frac{u_{T}-\bar{W}_{x}}{w_{T}-w_{\text {core }}} \\
& l_{x}=\frac{v_{T}-\bar{W}_{y}}{w_{T}-w_{\text {core }}},
\end{aligned}
$$

and the streamlines are defined by:

$$
\begin{aligned}
\Delta x^{\prime} & =\Delta x-l_{x} \Delta z \\
\Delta y^{\prime} & =\Delta y-l_{y} \Delta z \\
d_{H}^{\prime} & =\sqrt{\Delta x^{\prime 2}+\Delta y^{\prime 2}} \\
d_{H}^{\prime} & =r_{\text {max }}-\Delta z \tan \zeta^{\prime} \\
\Delta z & =\left(d_{H}^{\prime}-r_{\text {min }}\right) \tan \zeta^{\prime} \\
\frac{R_{T}^{2}}{r_{\text {min }}}+r_{\text {min }} & =\frac{\Delta z^{2}+d_{H}^{\prime 2}+R_{T}^{2}}{d_{H}^{\prime}} .
\end{aligned}
$$




\subsubsection{Thermal Models Comparison}

We now summarize the main similarities and differences among the presented thermal models. The models described above have some important similarities - the updraft speed is maximized at the thermal core and the updraft field presents a bell-like shape. The main differences among the models are the manner in which the flow field changes with altitude, the main parameters governing the bell-like shape of the updraft speed, the dependencies on the thermal outer radius, the thermal core movement, and how the thermal flow field leans with the wind. Table 4 summarizes these differences among models, which are explained in more detail next.

Table 4: Thermal models comparison

\begin{tabular}{|c|c|c|c|c|c|c|c|c|c|}
\hline Model & Dim & \multicolumn{2}{|c|}{ Downdraft } & \multicolumn{2}{|c|}{ Flat core } & \multicolumn{2}{|c|}{ Mix. layer ${ }^{\star}$} & \multicolumn{2}{|c|}{ Type } \\
\hline $\begin{array}{l}\text { Toroidal [3] } \\
\end{array}$ & 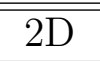 & \multicolumn{2}{|c|}{ No } & \multicolumn{2}{|c|}{ " Yes } & \multicolumn{2}{|c|}{ 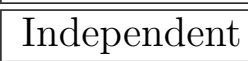 } & \multicolumn{2}{|c|}{ No type } \\
\hline Gaussian [1] & $2 \mathrm{D}$ & \multicolumn{2}{|c|}{ No } & \multicolumn{2}{|c|}{ No } & \multicolumn{2}{|c|}{ Independent } & \multicolumn{2}{|c|}{ No type } \\
\hline Gedeon $[2]$ & $2 \mathrm{D}$ & \multicolumn{2}{|c|}{ Constrained $^{3 \star}$} & \multicolumn{2}{|c|}{ No } & \multicolumn{2}{|c|}{ Independent } & \multicolumn{2}{|c|}{ No type } \\
\hline Allen [3] & $3 \mathrm{D}$ & \multicolumn{2}{|c|}{ Extended $^{4 \star}$} & \multicolumn{2}{|c|}{ Yes } & \multicolumn{2}{|c|}{ Dependent } & \multicolumn{2}{|c|}{ Chimney } \\
\hline Bencatel Mov. Chimn. ${ }^{6 \star}$ & $3 \mathrm{D}$ & \multicolumn{2}{|c|}{ Extended $^{4 \star}$} & \multicolumn{2}{|c|}{ Yes } & \multicolumn{2}{|c|}{ Dependent } & \multirow{2}{*}{\multicolumn{2}{|c|}{$\begin{array}{c}\text { Chimney } \\
\text { Bubble }\end{array}$}} \\
\hline Lawrance [4] & $3 \mathrm{D}$ & \multicolumn{2}{|c|}{ Constrained $^{3 \star}$} & \multicolumn{2}{|c|}{ No } & \multicolumn{2}{|c|}{ Independent } & & \\
\hline 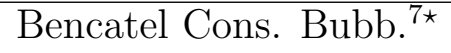 & $3 \mathrm{D}$ & \multicolumn{2}{|c|}{ Extended $^{4 \star}$} & Choic & & \multicolumn{2}{|c|}{ Independent } & & ibble \\
\hline Model & \multicolumn{2}{|c|}{ Flow Field $^{2 \star}$} & \multicolumn{2}{|c|}{ Conservative } & \multicolumn{2}{|c|}{ Leaning } & \multicolumn{2}{|c|}{ Movement } & \\
\hline Toroidal [3] & \multicolumn{2}{|c|}{ Vertical } & \multicolumn{2}{|c|}{ No } & \multicolumn{2}{|c|}{ "No } & $\mathrm{No}$ & & \\
\hline Gaussian [1] & & tical & $\overline{\mathrm{N}}$ & & & To & No & & \\
\hline Gedeon $[2]$ & & tical & $\mathrm{N}$ & & & Jo & No & & \\
\hline Allen [3] & & tical & $\mathrm{Y}$ & & & Jo & No & & \\
\hline Bencatel Mov. Chimn. ${ }^{6 \star}$ & & tical & $\overline{\mathrm{Y}}$ & & & es & Yes & & \\
\hline Lawrance [4] & & $\mathrm{D}$ & $\mathrm{N}$ & & & Jo & No & & \\
\hline Bencatel Cons. Bubb. ${ }^{7 \star}$ & & $\mathrm{D}$ & $\mathrm{Y}$ & & & es & Yes & & \\
\hline
\end{tabular}

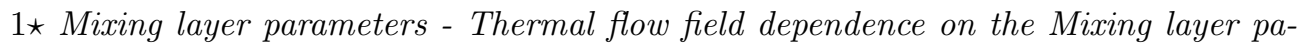
rameters.

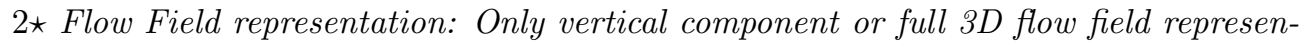
tation.

$3 \star$ Downdraft constrained to the thermal rim.

$4 \star$ Downdraft extended to the thermal surrounding area, beyond the thermal rim. 
$5 \star$ The user can choose if there is a flat plateau and/or the flatness/abruptness of the whole bell-shape updraft.

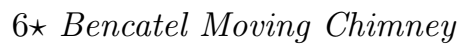

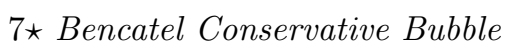

Altitude handling - Both Chimney Thermal and Bubble Thermal models incorporate variation with altitude. By contrast, the Toroidal, the Gaussian, and the Gedeon models are invariant with altitude, as shown in figure 30. The Allen model simulates a Chimney type thermal, where the thermal extends from the ground to the top of the mixinglayer (fig. 30a). In figure 30a it is possible to see the updraft speed and outer radius dependence over the altitude range. In the Bubble Thermal models the updraft region is limited to the mixing-layer, but may not reach either the ground or the top of the mixing layer (fig. 30b). In the Allen Chimney Thermal model, the updraft speed depends on the altitude. By contrast, the Lawrance Bubble Thermal model defines a fixed outer radius. The conservative Bubble Thermal models present a variable outer updraft radius, but this variation is distinctly different from the one defined by the Allen model.

Bell-like shape - All models present a Bell-like shape with some differences. The Allen Chimney Thermal model (fig. 31a) presents an updraft core with skirt downdrafts. In volumetric terms, the updraft and downdraft cancel each other, presenting a conservative airmass flow. Further, it shows a flat core for large diameter thermals. This shape is based on real observations as described by Irving [21]. The maximum updraft is not constant, decreasing as the outer diameter increases. The Lawrance Bubble Thermal model (fig. 31b) also presents a central updraft and exterior downdrafts. Unlike the downdrafts from the other models, in this model the negative vertical flow is constrained to a distance twice the outer radius. Moreover, the flow is only conservative at the bubble mean altitude, no flat core is modeled, and the maximum updraft only varies with altitude and not with the outer diameter. The conservative Bubble Thermal models present a mass conservative flow, with the downdraft extending to infinity, but decreasing with the distance to the bubble center. The core updraft also changes with altitude, although it covers different radii for both models. The conservative Bubble Thermal model with a Lawrance updraft field (fig. 31c) shows 
no flat core, while the one with the Plateau Updraft (fig. 31d) defines a near flat core. The Toroidal, the Gaussian-shape and the Gedeon models are simplifications which present the core updraft. The only difference between the Gaussian-shape and the Gedeon models is the presence of a skirt downdraft on the last. These three models are not flow conservative and their maximum updraft is always constant, with respect to the altitude and outer diameter. 


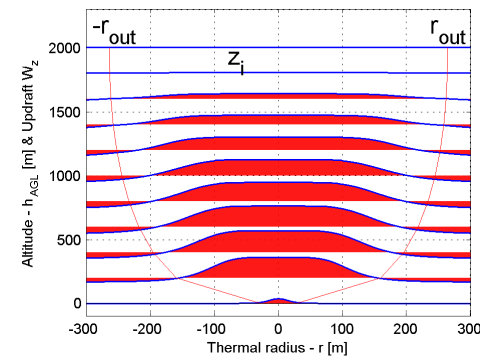

(a) Allen Chimney Thermal model - The outer diameter varies with the altitude as well as with the updraft speed.

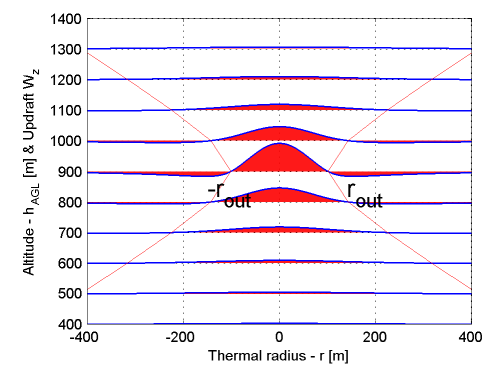

(c) Conservative Bubble Thermal model, with Lawrance updraft field - The outer diameter varies with the altitude as well as with the updraft speed.

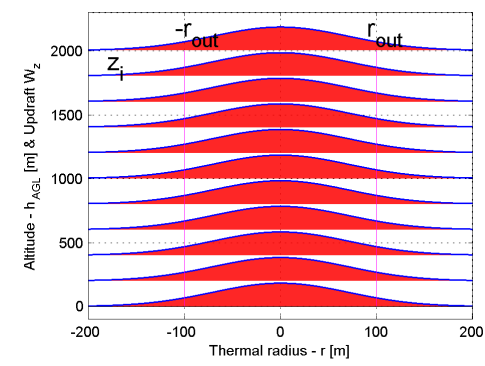

(e) Gaussian Thermal model - The altitude doesn't affect the outer radius or the updraft speed.

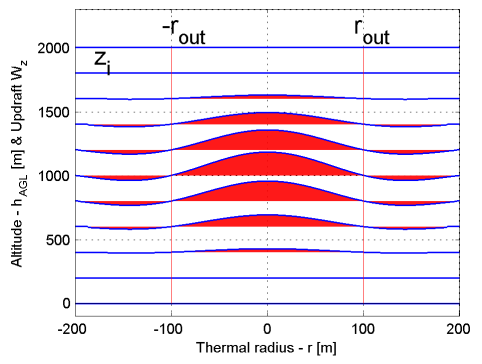

(b) Lawrence Bubble Thermal model - The updraft speed varies with the altitude, but not with the outer diameter.

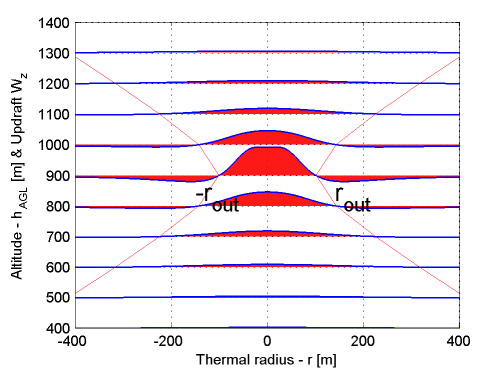

(d) Conservative Bubble Thermal model, with Plateau Updraft field The outer diameter varies with the altitude as well as with the updraft speed.

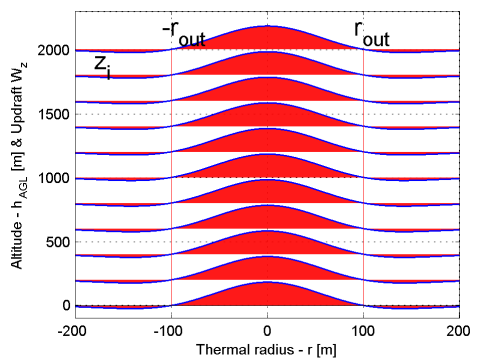

(f) Gedeon Thermal model - The altitude doesn't affect the outer radius or the updraft speed.

Figure 30: Differences on altitude dependence of the different models. 
Outer radius - For most of the models the outer radius represents the distance to the core where the vertical flow is inverted, turning from updraft to downdraft. That is the case for the Bubble Thermal models, the Toroidal model, and the Gedeon model. Further, in the Bubble Thermal models the outer radius at the Bubble mean altitude also indicates the center for the toroid vortex. Both the Allen and the Conservative Bubble Thermal models have the mean updraft strength as a function of the outer radius, and outer radius a function of the altitude. In the Allen model the flow field at the outer radius is almost null, but not quite. In the Gaussian model the outer radius is just a measure of the radial spread of the updraft. 


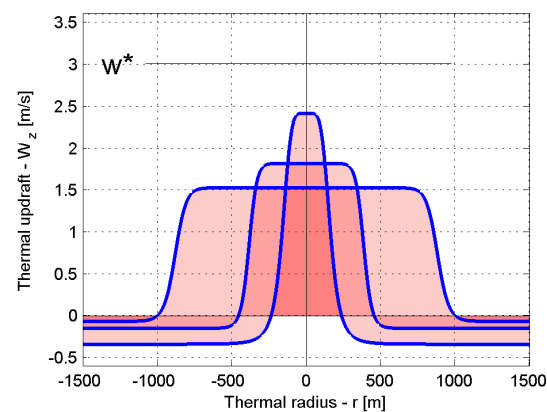

(a) Allen Chimney Thermal model Presents a constant updraft speed at the core for large diameter thermals. Further, the updraft speed is inversely correlated with the thermal diameter.

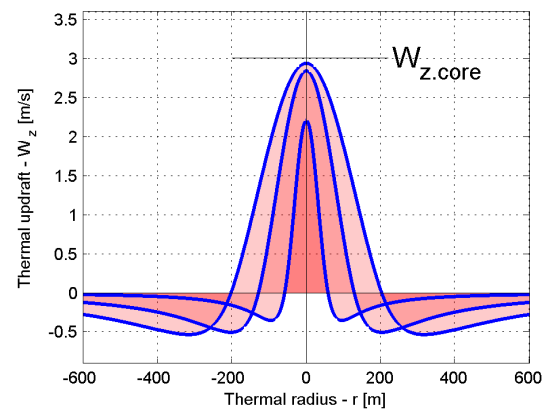

(c) Conservative Bubble Thermal model, with Lawrance updraft field - Presents a correlation between the updraft speed and the thermal diameter.

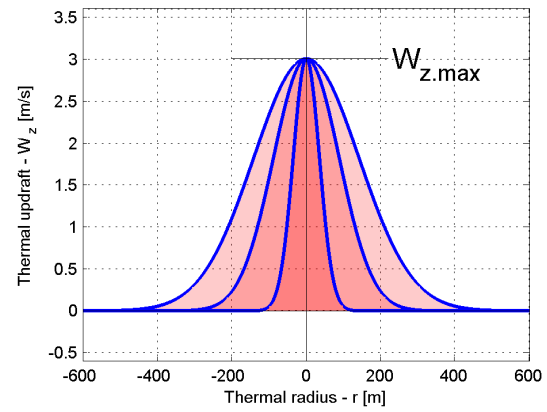

(e) Gaussian Thermal model - Presents a constant maximum updraft speed and no flat core for large diameter thermals.

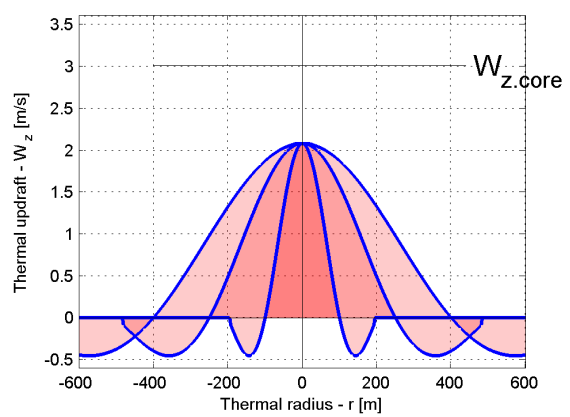

(b) Lawrance Bubble Thermal model Presents a maximum updraft speed constant with the altitude and no flat core for large diameter thermals.

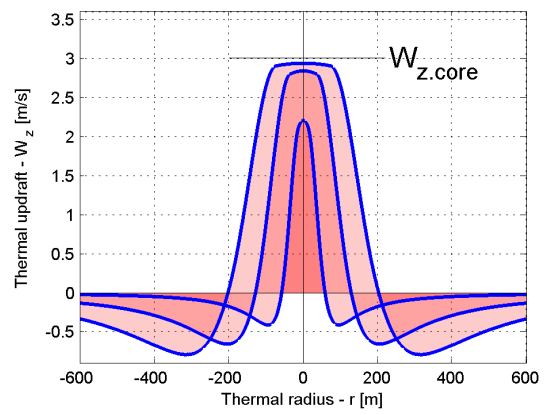

(d) Conservative Bubble Thermal model, with Plateau updraft field Presents an almost constant updraft speed at the core for large diameter thermals and a correlation between the updraft speed and the thermal diameter.

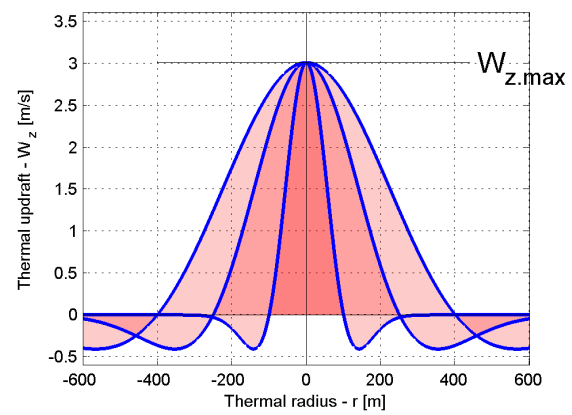

(f) Gedeon Thermal model - Presents a 7 constant maximum updraft speed and no flat core for large diameter thermals.

Figure 31: Updraft function shape among the different models. 


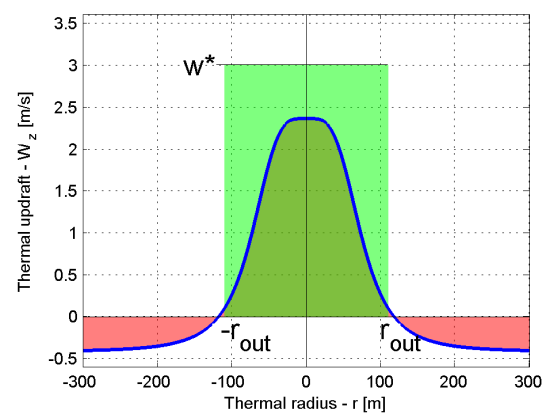

(a) Allen Chimney Thermal model.

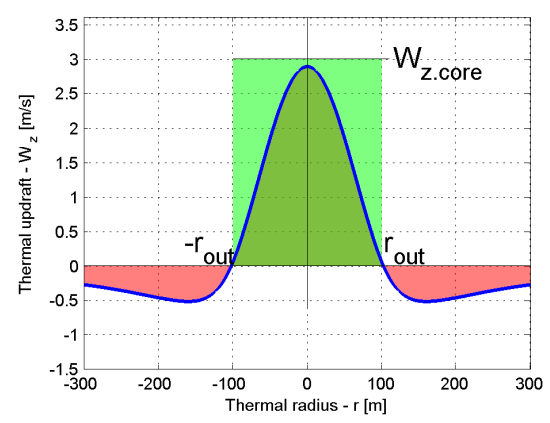

(c) Conservative Bubble Thermal model, with Lawrance updraft field.

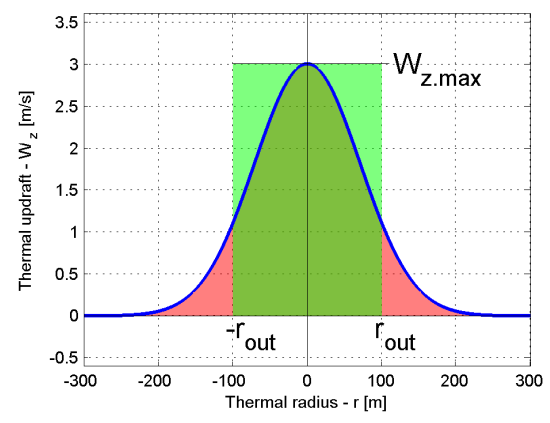

(e) Gaussian Thermal model.

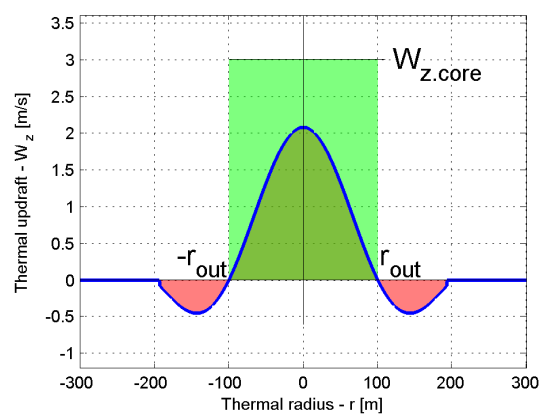

(b) Lawrance Bubble Thermal model.

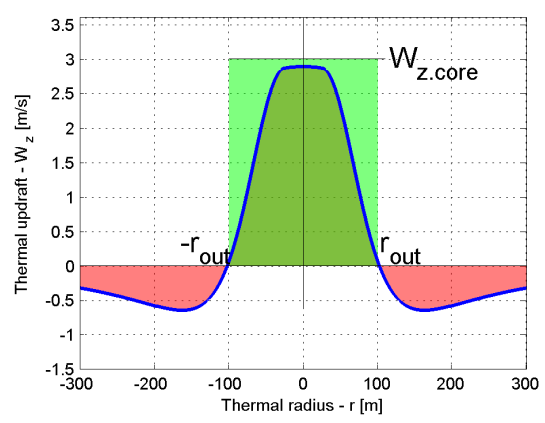

(d) Conservatibe Bubble Thermal model, with Plateau updraft field.

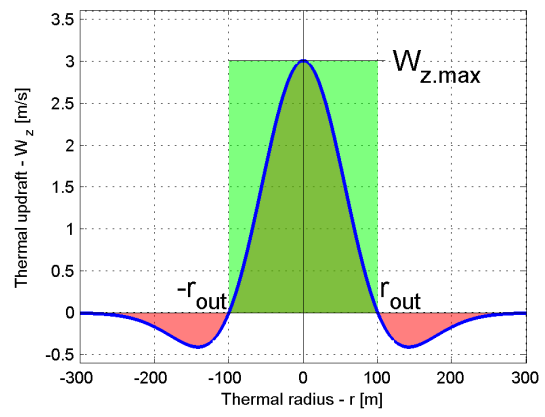

(f) Gedeon Thermal model

Figure 32: Differences in outer radius dependence of the different models. The outer radius presents a null updraft speed for most of the models. The exceptions are the Allen Chimney Thermal model, where the updraft is almost null at the outer radius, and the Gaussian model, which always presents a positive updraft speed, about $37 \%$ of the maximum updraft speed at the outer radius. 
We may extend the Gaussian and the Gedeon models into more realistic Chimney models, if we use the Allen model's outer radius function (22) and core updraft function (26). This would make the outer radius a function of altitude and the updraft speed a function of both altitude and outer radius (fig. 33). Even so, these models would remain non conservative in terms of air mass exchange.

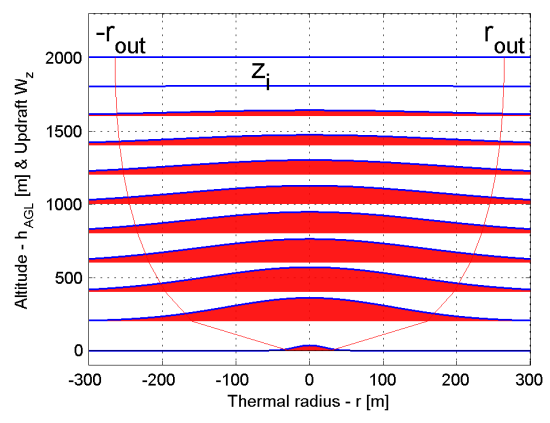

(a) Allen-Gaussian Thermal model

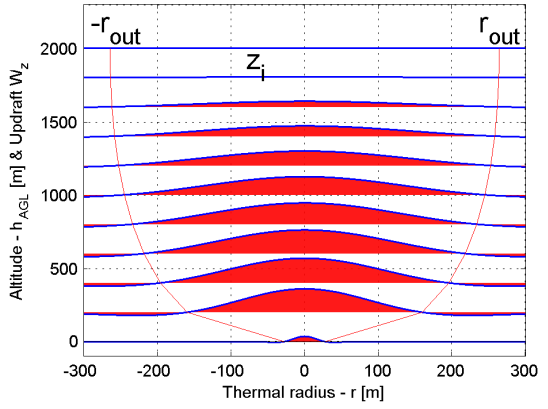

(b) Allen-Gedeon Thermal model

Figure 33: Adaptation of the Gaussian shape and Gedeon models to simulate a Chimney type thermal - The altitude affects the outer radius and the maximum updraft speed in the same manner as in the Allen model.

\subsection{Thermals Development and Fading}

Individual thermals' appearance rate may be modeled by a Poisson distribution. Based on Lenschow and Stephens' data collected over the ocean [20], the average number of thermals encountered over a path of length $s$, normalized by the Mixed-Layer altitude $z^{i}$, is

$$
\overline{N_{T}} \approx \frac{1.2 s}{z^{i}}, z \in\left[0.1 z^{i}, z^{i}\right]
$$

where $z$ is the measurement altitude. As such, the resulting Poisson distribution is

$$
P_{\text {Thermal }}(s)=1-e^{-\lambda_{T} \cdot s}, \lambda_{T} \approx \frac{1.2}{z^{i}} .
$$

Thermals present different strengths and sizes, even if they are developed in the same region with the same environmental conditions. The size probability distribution of an ensemble of thermals follows a Gamma distribution [22, 23]. Vulf'son and Borodin derived analytically the distribution 
of spontaneous convective jets and compared the results with several sets of empirical data. First they derived an invariant relation between the thermal diameter and its average vertical velocity, at each level h:

$$
\hat{w}=\frac{3 g \theta_{T}}{16 \alpha_{R}} D .
$$

where $g$ is the gravity acceleration, $\theta_{T}$ is the environment temperature excess and $\alpha_{R}=0.1$. The size and strength probability density function (pdf) was then defined as:

$$
\begin{aligned}
& \frac{N_{w}}{N_{0}}=\frac{a}{\Gamma(a) \overline{\hat{w}^{2}}}\left[\frac{a \hat{w}^{2}}{\hat{\hat{w}}^{2}}\right]^{a-1} e^{\frac{-a \hat{\omega}^{2}}{\overline{\hat{w}}^{2}}} \\
& \frac{N_{D}}{N_{0}}=\frac{a}{\Gamma(a) \bar{D}}\left[\frac{a D}{\bar{D}}\right]^{a-1} e^{\frac{-a D}{\bar{D}}}
\end{aligned}
$$

where $\overline{\hat{w}^{2}}$ and $\bar{D}$ are the average strength and diameter among all thermals, $N_{w} / N_{0}$ and $N_{D} / N_{0}$ are the fraction of thermals with strength or diameter in the interval $\left[\hat{w}^{2},(\hat{w}+d \hat{w})^{2}\right]$ or $[D, D+d D], N_{0}$ is the total number of thermals, and $a$ is the pdf shape parameter (fig. 34). The pdf differs greatly from the surface layer to the rest of the Mixed-Layer. The surface layer extends from the ground to 100-300 meters. At these altitudes the size distribution is characterized by $a-1=1.67$, while in the above layer the distribution shape is characterized by $a-1=2.13[22,23]$.

A thermal may fade after some time or merge with nearby thermals, forming a bigger thermal that will itself fade away after some time. Thermals' lifespan can range from 5 to 30 minutes $[17,3,24]$, with 20 minutes as the mean lifespan.

\subsection{Experimental Verification of Thermal Flow Field Models}

An important issue in models is their verification as representative of the reality. In the case of thermals and orographic updrafts one of the most important features is their internal flow field structure. We have not found any study referring to airflow data collected inside thermals or orographic updrafts that could be useful for the validation of these features. The studies we found referring to real data from thermals are from Lenschow and Stephens [20] and from Allen [3]. 
Allen [3] studied the distribution of two environmental parameters that influence the thermal size and strength, in Desert Rock, Nevada. These parameters are the maximum altitude a thermal may reach, i.e., the atmosphere mixing-layer altitude, and the convection intensity in the mixing-layer $\left(z_{i}\right)$, represented by the convective velocity scale $\left(w^{\star}\right)$, which is related to the thermals' updraft speed.

Lenschow and Stephens's work [20] provides the best data to relate the altitude with the average thermal horizontal size and the updraft speed. The data was collected from a thermal field over the ocean, flying long lines and averaging the airflow data among all detected thermals at each altitude. This method is good to obtain the general relations between altitude and thermal size and strength. However, it is not good enough to evaluate the evolution of the thermal diameter within individual thermals. That would be important to evaluate the 3D shape of the thermals, identifying them as Chimney or Bubble Thermals and allowing the validation of the models presented in section 4.1. Furthermore, the averaging of the thermal airflow observations makes it impossible to validate the internal thermal flow field predicted by any thermal model.

\subsection{Orographic Updrafts}

Ridge lift - Ridge or slope lift appears along the windward side of mountain ridges. It is generated when the flowing air mass collides with the mountain side and is forced to climb to overcome the obstacle. Langelaan described a general model for the ridge flow field based on a semi-cylindric obstacle over a flat surface [25] (fig. 35):

$$
\begin{aligned}
\mathbf{w} & =w_{\infty} \hat{\mathbf{x}}-w_{\infty} \frac{R^{2}}{r^{2}}(\cos \eta \hat{\mathbf{r}}+\sin \eta \hat{\eta}) \\
w_{x} & =w_{\infty}\left[1-\frac{R^{2}}{r^{2}}\left(\cos ^{2} \eta-\sin ^{2} \eta\right)\right] \\
w_{z} & =2 w_{\infty} \frac{R^{2}}{r^{2}} \cos \eta \sin \eta,
\end{aligned}
$$

The updraft is generated if the general wind direction is within $30^{\circ}$ to $40^{\circ}$ to the perpendicular to the ridge line [13]. Unlike thermals, the ridge updraft maintains a stable position, endures as long as the wind conditions are favorable, and can cover large areas, allowing long upward soaring legs. The main limitation of the ridge lift is that it is constrained to a limited altitude above the ridge crest. 
Wave lift - Wave lift is another effect of mountainous terrain. It develops after the air mass has passed over the undulating terrain at high altitudes. "This lift is part of a large scale deflection of air mass, which is known as "lee wave" lift, first recognized in the 1930s and explored scientifically in the early 1950s" [26]. The lee wave flow field structure is similar to a sinusoidal wave (fig. 35b). "Lee wave field structures the air mass sink rates in parallel bands having high cross-stream coherence" [27]. The wave length $\lambda_{W}$ of this phenomenon depends on the atmospheric conditions, in particular the atmosphere stability. "In the atmosphere, ... $\lambda_{W} \sim \mathcal{O}(10$ n.mi.) for hydrostatic lee waves or $\lambda_{W} \sim \mathcal{O}$ (1n.mi.) for nonhydrostatic lee waves" [27]. Wave lift development depends greatly on the presence of relatively high winds and stable atmosphere conditions.

\section{Wind Gust Models}

Among the studied phenomena in this work, gusts are the one most commonly encountered by Aircraft. But, unlike wind shear and updrafts reviewed above, the wind gusts are a very short term phenomenon. This and the difficulty to predict gusts' appearance makes them a lot harder to use for energy harvesting. For the sake of completeness, we include here a short review of gust models.

Gusts may convey energy to an aircraft in a similar manner to the wind shear phenomena. This energy is present in the wind velocity and direction variations, i.e., airflow gradients [4, 2].

\subsection{Gaussian Gust Model}

Several models are used to simulate gust turbulence. The simplest model is a Gaussian noise model, where the mean wind is complemented by deviations generated from a Gaussian distribution:

$$
\begin{aligned}
W_{x} & =\bar{W}_{x}+\Delta W \cos \psi_{G} \\
W_{y} & =\bar{W}_{y}+\Delta W \sin \psi_{G} \\
\Delta W & \sim \mathcal{N}\left(0, k_{G}\|\bar{w}\|\right) \\
\psi_{G} & \sim \mathcal{U}(0, \pi),
\end{aligned}
$$

where $\bar{W}_{x}$ and $\bar{W}_{y}$ are the mean wind velocities. $\Delta W$ is the total velocity variation and $\psi_{G}$ is the velocity variation direction; they are generated by 
the Normal distribution $\mathcal{N}\left(0, k_{G}\|\bar{w}\|\right)$, with standard deviation proportional to the total wind speed $\sigma_{G}=k_{G}\|\bar{w}\|$, and the Uniform distribution $\mathcal{U}(0, \pi)$.

\subsection{Gauss-Markov Gust Model}

A more realistic model is the Gauss-Markov process random noise model. In this model each generated wind deviation doesn't only affect the wind at the generation moment, but its effect is also extended to the next moments by a decaying function. This yields a smoother and also more realistic wind variation. The deviations are still generated from a Gaussian distribution, but those are added to a decaying function from past deviations:

$$
\begin{aligned}
W_{x} & =\bar{W}_{x}+\Delta W_{x} \\
W_{y} & =\bar{W}_{y}+\Delta W_{y} \\
\Delta W_{x} & =\Delta W_{x}^{\prime}\left(1-\frac{\Delta t}{\tau_{G}}\right)+\Delta W^{+} \cos \psi_{G+} \\
\Delta W_{y} & =\Delta W_{y}^{\prime}\left(1-\frac{\Delta t}{\tau_{G}}\right)+\Delta W^{+} \sin \psi_{G+} \\
\Delta W^{+} & \sim \mathcal{N}\left(0, k_{G+}\|\bar{w}\|\right) \\
\psi_{G+} & \sim \mathcal{U}(0, \pi),
\end{aligned}
$$

where $\bar{W}_{x}$ and $\bar{W}_{y}$ are the mean wind velocities. $\Delta W_{x}$ and $\Delta W_{y}$ are the wind variation velocities. $\Delta W_{x}^{\prime}$ and $\Delta W_{y}^{\prime}$ are the past wind variation velocities. $\tau_{G}$ is the decay time constant. $\Delta W^{+}$is the total velocity extra variation and $\psi_{G+}$ is the velocity extra variation direction; they are generated by the Normal distribution $\mathcal{N}\left(0, k_{G+}\|\bar{w}\|\right)$, with standard deviation proportional to the total wind speed $\sigma_{G+}=k_{G+}\|\bar{w}\|$, and the Uniform distribution $\mathcal{U}(0, \pi)$. The extra wind variation standard deviation $\left(\sigma_{G+}\right)$ is computed by the combination of the decay time constant $\left(\tau_{G}\right)$ and the overall wind standard deviation $\left(\sigma_{G}\right)$ :

$$
\sigma_{G+}=\sigma_{G} \sqrt{\frac{2}{\tau_{G}}} .
$$

\subsection{Dryden and von Karman Gust Models}

The Military Specification MIL-F-8785C [6] defines three gust models. The simplest model is the "1-cosine" shape model. The more realistic and

most accepted models for aircraft simulation are the von Karman and the Dryden gust fields. 


\subsection{Urban Gust Models}

The gust models presented above are well suited for open area flow simulation. However they don't capture complex flow environments well, e.g., a flow field in an urban grid. Models with more detail simulate those kind

of environments better. For that, Cybyk et al [28] implemented a real-time physics-based simulation tool to study UAV dynamics in a urban environment.

\subsection{Wind Gust Models Comparison}

We now compare the presented gust models. Table 5 highlights the differences between the models.

Table 5: Wind Gust Models Comparison

\begin{tabular}{|c|c|c|c|c|}
\hline Model & Comp. Load ${ }^{1 \star}$ & Pwr Spectrum ${ }^{2 \star}$ & Grad. Fid. ${ }^{3 \star}$ & Obj. Interaction ${ }^{4 \star}$ \\
\hline Gaussian & Low & No & Low & No \\
\hline Gauss-Markov & Low & No & Medium & No \\
\hline Dryden & Medium & Yes & High & $\mathrm{No}$ \\
\hline von Karman & Medium & Yes & High & No \\
\hline Urban & High & Maybe & High & Yes \\
\hline
\end{tabular}

$1 \star$ Computational load required to predict the flow field.

$2 \star$ Gusts appearance power spectrum.

$3 \star$ Flow field gradient fidelity, e.g., airflow velocity variation smoothness.

$4 \star$ Prediction of the interaction effects between the flow field and solid objects.

From table 5 we can conclude that the Gauss-Markov model should be preferred for simpler simulations, in particular, simulations where the gust appearance power spectrum is not a paramount factor. The Gaussian gust model should only be used if the available computation power is very limited.

We may also conclude from table 5 that the Dryden or the von Karman models should be used, if we need to simulate a gust field with a correct distribution in the frequency domain. Finally, if we need to predict complex gust fields interacting with objects, it is required to use a model like the urban gust simulation, such as the one presented by Cybyk et al [28]. 


\section{Conclusions}

This survey lays out the basis for the development of flow field energy harvesting by aircraft. This work studies an array of atmospheric flow field phenomena selected by their perceived exploitation potential to extend aircraft flight endurance. We studied several existing models for thermal updrafts, wind shear and gusts.

Further, we improved some of those models and created new ones to better capture some important features. Examples of this are the improved Chimney Thermal model and the new Bubble Thermal, Layer Wind Shear, and Ridge Wind Shear models.

The studied models represent the phenomena's flow field shape in 1D, $2 \mathrm{D}$ or $3 \mathrm{D}$, the interaction with the steady wind flow, and the dynamics. For thermals we presented general 2D models, and 3D models for Chimney and Bubble Thermals. The Bubble Thermal model is new and is based on the vortex shell hypothesis by Cone [14]. The extended Chimney Thermal model is based on the Allen model [3], and includes the thermal core movement and thermal interactions with the surrounding flow field, e.g., its leeward leaning when there is wind. In terms of wind shear we presented existing models for general wind shear and the Surface Wind Shear, and developed new models for the Layer and the Ridge Wind Shear. For completeness, we closed this survey with a short review of gusts models.

\section{Future Work}

Most of the models presented in this work are based on the observation of bird flight and glider pilots observations. An important extension of this work will be the validation of the models through the measurement of the corresponding flow fields. We plan to use a team of UAV flying in formation [29] to simultaneously collect air velocity data from several points in space. This data will then be compared to the model predictions to assert the fidelity of the models.

The flow field phenomena models presented in this work will support the development of estimators for the flow field phenomena. The combination of simpler models and more realistic ones will allow the implementation of low computational load estimators. These should use the simpler models to provide the initial convergence. Once an initial rough estimate exists, the higher fidelity models will allow the estimators to distinguish between phenomena types and provide more precise tracking and predictions. 
The presented models will also serve to simulate UAVs' flight through flow field phenomena. Further, the flow field models will be the basis for the definition of flight controllers tailored for energy harvesting.

\section{Acknowledgments}

We are specially grateful to Professor Pierre Kabamba, from the University of Michigan Aerospace Department, who carefully reviewed this manuscript and provided precious insights. We gratefully acknowledge the support of the Aerospace \& Robotic Controls Laboratory researchers, in particular Justin Jackson, Baro Hyun, Mariam Faied, and Zahid Hasan, at the University of Michigan. We further acknowledge the support of the AsasF group and the researchers from the Underwater Systems and Technology Laboratory, specially Filipe Ferreira, Joel Cardoso, Sérgio Ferreira, Joel Gomes, Daniel Silva, Paulo Lopes, and Gil Gonçalves, and the Portuguese Air Force Academy, specially Cap. Elói Pereira, Lt. Tiago Oliveira, Lt. Gonçalo Cruz, Sgt. Paulo Teixeira, Sgt. Joaquim Gomes, and Cap. Paula Gonçalves. The research leading to this work was funded in part by the United States Air Force under grant number FA 8650-07-2-3744, by Financiamento pluri-anual of the FEUP ISR Porto R\&D unit, and by the FCT (Foundation for Science and Technology) under PhD grant SFRH/BD/40764/200\%.

\section{References}

[1] Wharington J. Autonomous control of soaring aircraft by reinforcement learning. Ph.D. thesis; Royal Melbourne Institute of Technology, Faculty of Engineering, Department of Aerospace Engineering; 1998.

[2] Langelaan J. Gust energy extraction for mini and micro uninhabited aerial vehicles. Journal of Guidance, Control, and Dynamics 2009;32(2):464-73. doi:10.2514/1.37735.

[3] Allen MJ. Updraft model for development of autonomous soaring uninhabited air vehicles. In: 44rd AIAA Aerospace Sciences Meeting and Exhibit. Reno, Nevada: AIAA; 2006,.

[4] Lawrance NR, Sukkarieh S. Wind energy based path planning for a small gliding unmanned aerial vehicle. In: AIAA Guidance, Navigation, and Control Conference. Chicago, Illinois: AIAA; 2009,. 
[5] Sachs G. Minimum shear wind strength required for dynamic soaring of albatrosses. Ibis - The International Journal of Avian Science 2005;147(1):1-10. doi:10.1111/j.1474-919x.2004.00295.x. URL http://dx.doi.org/10.1111/j.1474-919x.2004.00295.x.

[6] U.S. Military Specification MIL-F-8785C; 1980.

[7] Grenestedt JL, Spletzer JR. Optimal energy extraction during dynamic jet stream soaring. In: AIAA Guidance, Navigation, and Control Conference. Toronto, Ontario, Canada; 2010,.

[8] Sachs G, da Costa O. Dynamic soaring in altitude region below jet streams. In: AIAA Guidance, Navigation, and Control Conference and Exhibit. Keystone, Colorado; 2006,.

[9] Zhao YJ. Optimal patterns of glider dynamic soaring. Optimal Control Applications and Methods 2004;25(2). doi:10.1002/oca.739.

[10] Parle J. Preliminary dynamic soaring research using a radio control glider. In: 42nd AIAA Aerospace Sciences Meeting and Exhibit. Reno, Nevada, USA; 2004,.

[11] Sachs G, Traugott J, Holzapfel F. In-flight measurement of dynamic soaring in albatrosses. In: AIAA Guidance, Navigation, and Control Conference. Toronto, Ontario, Canada; 2010,.

[12] Sachs G, Traugott J, Holzapfel F. Progress against the wind with dynamic soaring: Results from in-flight measurements of albatrosses. In: AIAA Guidance, Navigation, and Control Conference and Exhibit. Portland, Oregon, USA; 2011,.

[13] FAA . Aviation Weather. Aviation Supplies \& Academics, Inc.; 1975. ISBN 1560270012. URL http://www.aviationweather.ws/.

[14] Cone CD. Thermal soaring of birds. American Scientist 1962;50(1):180209.

[15] Andersson K, Kaminer I. Stability of a thermal centering controller. In: AIAA Guidance, Navigation, and Control Conference. Chicago, Illinois: AIAA; 2009,. 
[16] Edwards DJ. Implementation details and flight test results of an autonomous soaring controller. In: AIAA Guidance, Navigation, and Control Conference. Honolulu, Hawaii: AIAA; 2008,.

[17] Stull RB. An Introduction to Boundary Layer Meteorology. Norwell, Massachusetts: Kluwer Academic Publishers; 1988. ISBN 90-277-27694 .

[18] Konovalov DA. On the structure of thermals. In: 12th OSTIV Congress. Alpine, USA: OSTIV; 1970,.

[19] Irving F. The paths of soaring flight. World Scientific Publishing Company; 2004.

[20] Lenschow DH, Stephens PL. The role of thermals in the convective boundary layer. Boundary-Layer Meteorology 1980;19(4):509-32. doi: 10.1007/BF00122351.

[21] Irving F. The paths of soaring flight. Imperial College Press; 1999. ISBN $1860940552,9781860940552$.

[22] Vul'fson AN, Borodin OO. Size distribution of convective thermals in an unstable stratified layer. In: 18th Mechanical French Congress. Grenoble, France; 2007,.

[23] Vul'fson AN, Borodin OO. The size distribution function for mixed-layer thermals in the convective atmosphere. In: Borisov AV, Kozlov VV, Mamaev IS, Sokolovskiy MA, Gladwell GML, Moreau R, editors. IUTAM Symposium on Hamiltonian Dynamics, Vortex Structures, Turbulence; vol. 6 of IUTAM Bookseries. Springer Netherlands. ISBN 978-1-40206744-0; 2008, p. 227-36.

[24] Allen MJ. Autonomous soaring for improved endurance of a small uninhabited air vehicle. In: 43rd AIAA Aerospace Sciences Meeting and Exhibit. Reno, Nevada: AIAA; 2005,.

[25] Langelaan JW. Long distance/duration trajectory optimization for small uavs. In: AIAA Guidance, Navigation, and Control Conference and Exhibit. Hilton Head, South Carolina, USA; 2007,. 
[26] Short S. Birth of american soaring flight: A new technology. AIAA Journal 2005;43(1):17-27. doi:10.2514/1.10390.

[27] Jenkins SA, Wasyl J. Optimization of glides for constant wind fields and course headings. Journal of Aircraft 1990;27(7):632-8. doi: $10.2514 / 3.25333$.

[28] Cybyk BZ, McGrath B, Frey TM, Drewry DG, Keane JF. Unsteady urban airflows and their impact on small unmanned air system operations. In: AIAA Guidance, Navigation, and Control Conference. Chicago, Illinois: AIAA; 2009,.

[29] Bencatel R, Faied M, Sousa J, Girard A. Formation control with collision avoidance. In: 50th IEEE Conference on Decision and Control and European Control Conference. Orlando, Florida, USA; 2011,. 


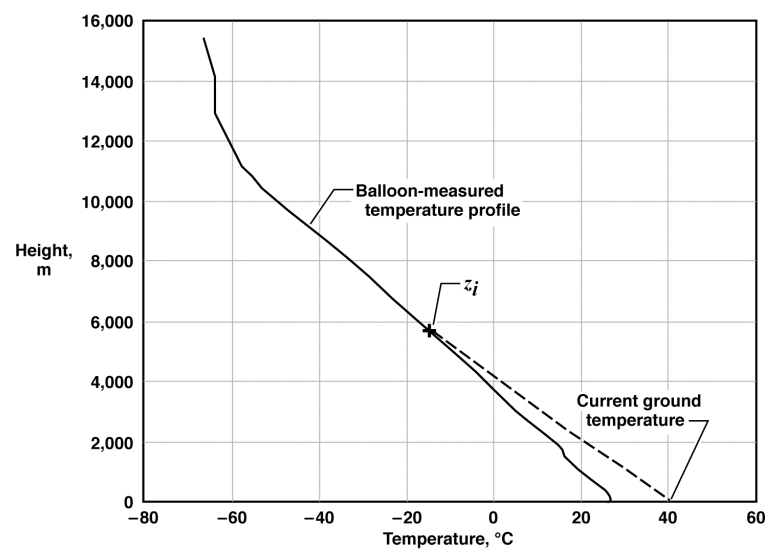

Figure 16: Example of $z_{i}$ calculation (illustration from [3])

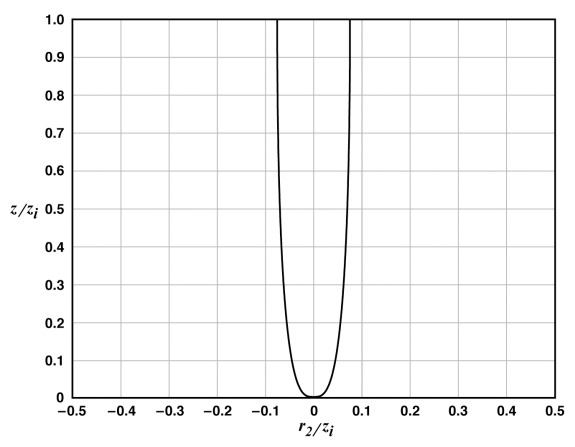

Figure 17: Updraft outer radius $\left(r_{2}\right)$ function of $z_{i}$ (illustration from [3])

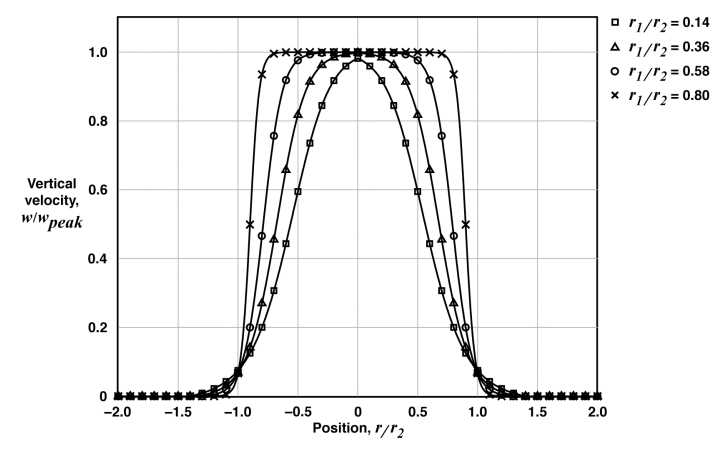

Figure 18: Updraft velocity bell-shape model (illustration from [3]) 


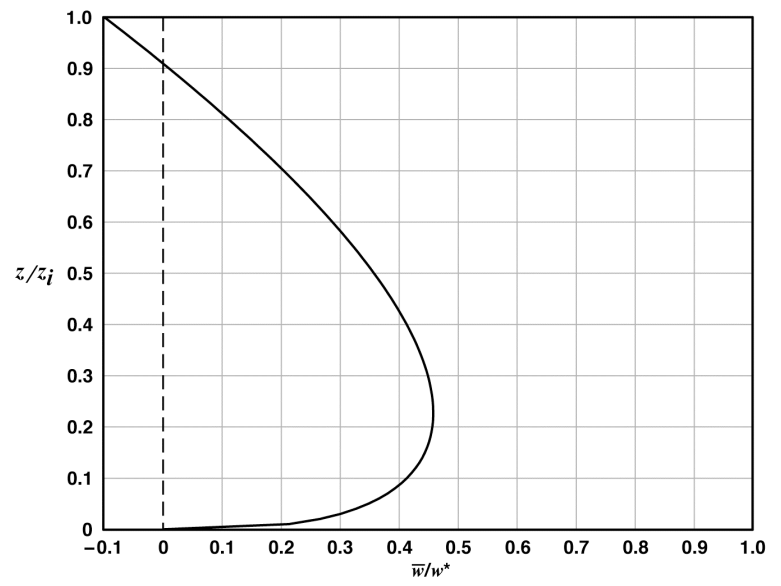

Figure 19: Average updraft velocity at different altitudes (illustration from [3])

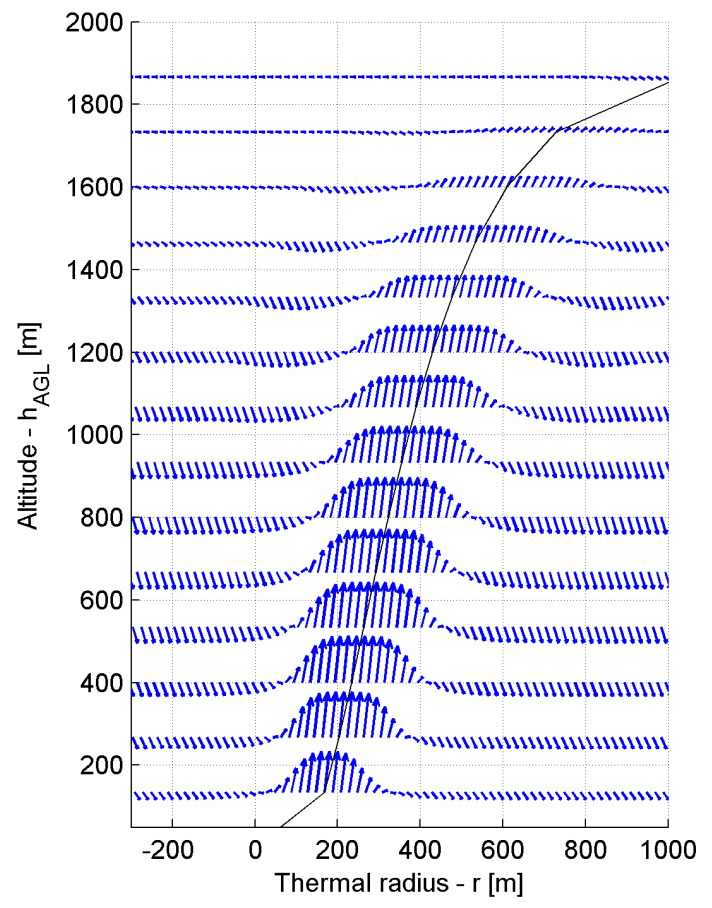

Figure 20: Leaning Chimney Thermals. Effect of wind on the shape of the thermal flow field. 


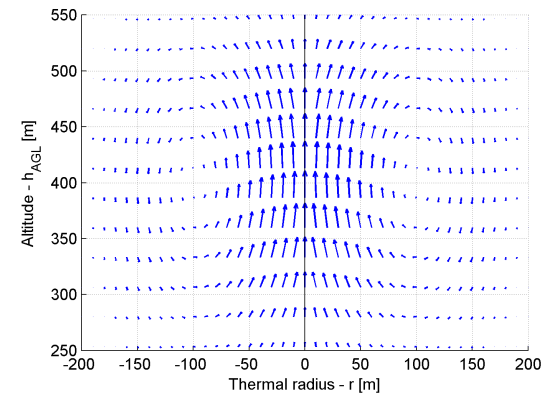

(a) 2D flow field

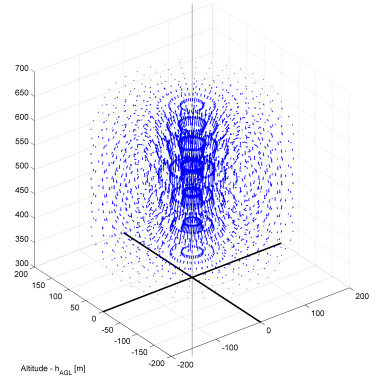

(b) 3D flow field

Figure 21: Flow field in a Lawrance Bubble Thermal. The vortex is noticeable around the thermal radius $(100 \mathrm{~m})$ at the mean altitude $(400 \mathrm{~m})$. 


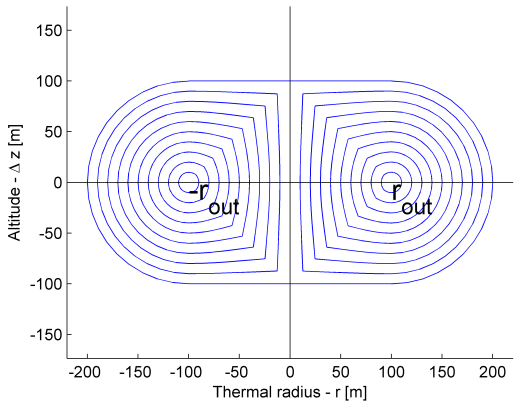

(a) $1^{\text {st }}$ streamline hypothesis. Presents sharp flow direction changes.

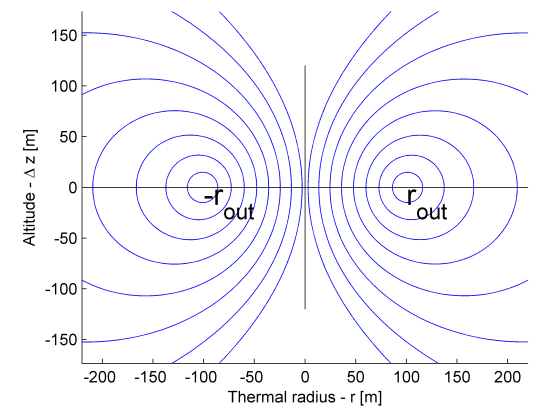

(c) $3^{\text {rd }}$ streamline hypothesis. Presents no flow near the bubble axis, when away from the central plane.

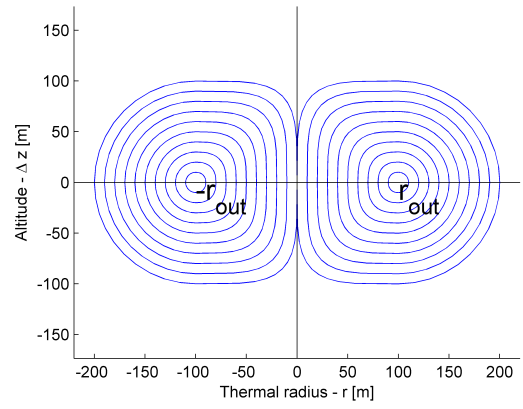

(b) $2^{\text {nd }}$ streamline hypothesis. Lacks any flow near the top and the bottom of the bubble.

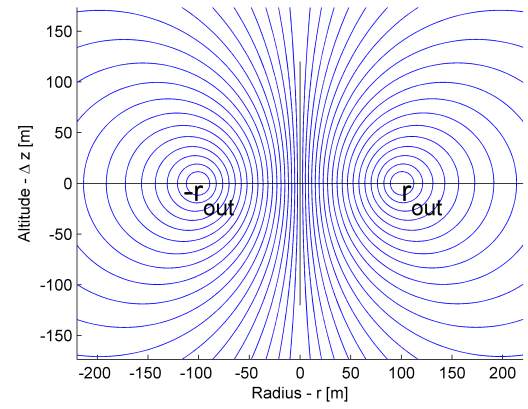

(d) Final streamline hypothesis.

Figure 22: Sequence of streamline models for a conservative flow Bubble Thermal model. 


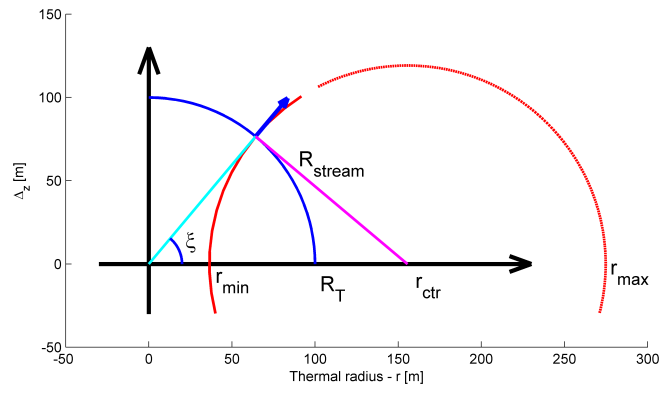

Figure 23: Streamline parameters definition.

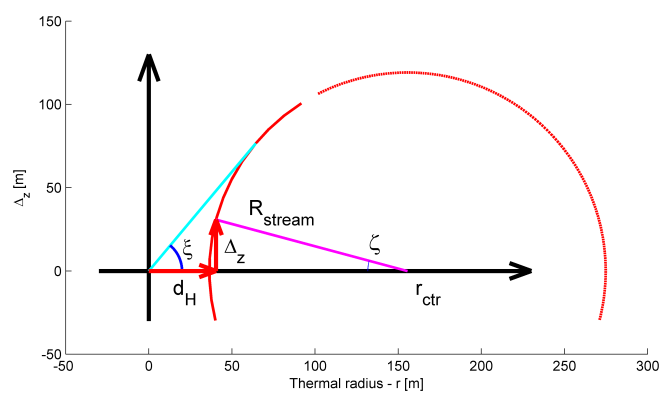

Figure 24: Random point definition on a streamline. 


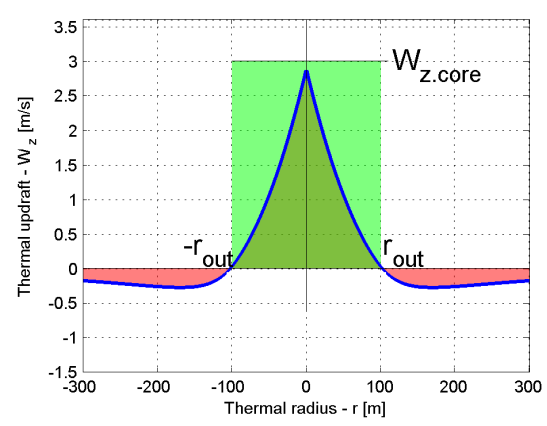

(a) Vertical flow field with $\delta_{\text {Flat }}=0.5$

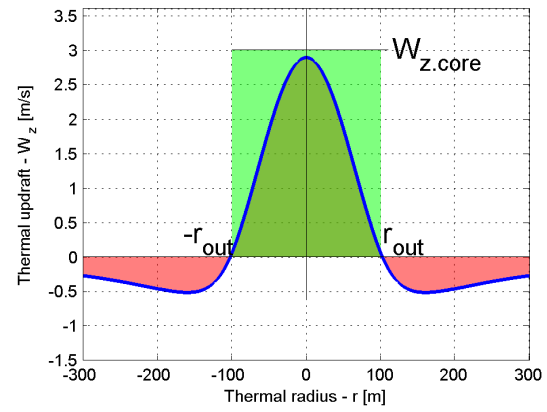

(c) Vertical flow field with $\delta_{\text {Flat }}=1$

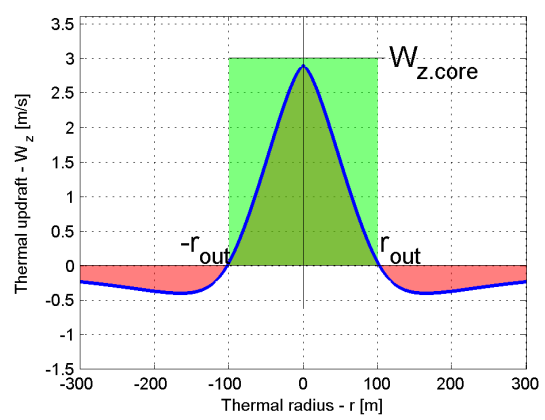

(b) Vertical flow field with $\delta_{\text {Flat }}=0.75$

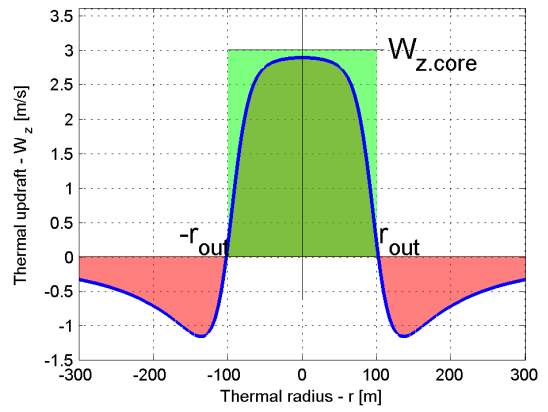

(d) Vertical flow field with $\delta_{\text {Flat }}=4$

Figure 25: Vertical flow field based on Lawrance updraft field, with an updraft outer radius of $100 \mathrm{~m}$. 


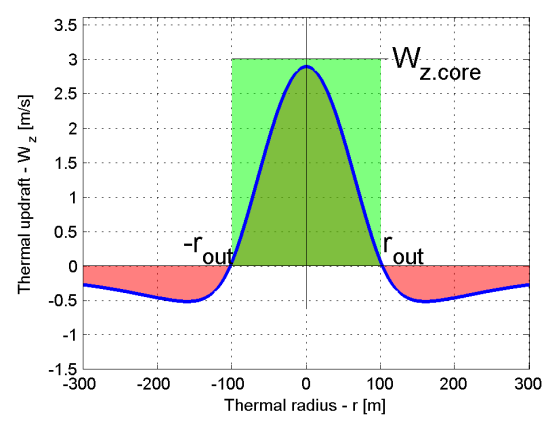

(a) Lawrance vertical flow field model

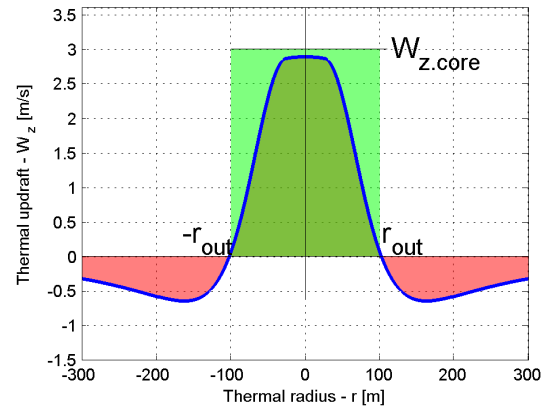

(c) Plateau Updraft vertical flow field model with $R_{T}=100$

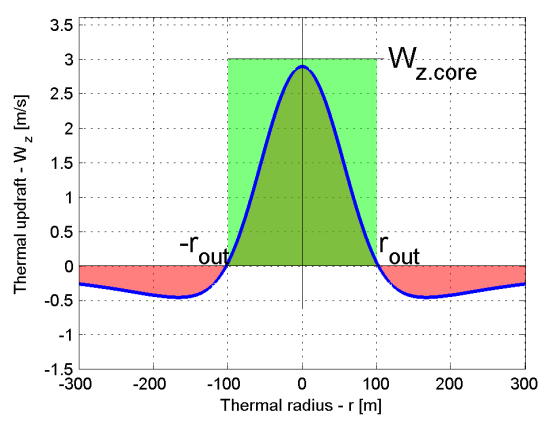

(b) Gedeon vertical flow field model

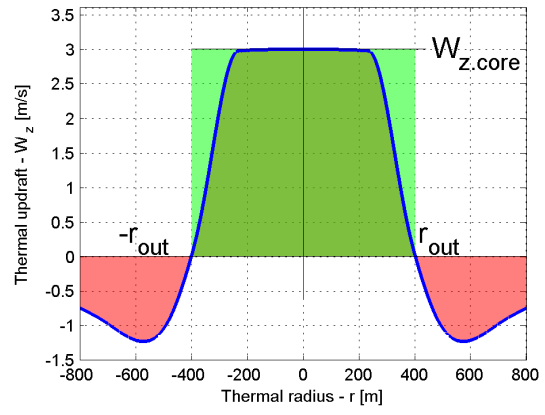

(d) Plateau Updraft vertical flow field model with $R_{T}=400$

Figure 26: Comparison between vertical flow field models. Lawrance and Gedeon updraft models yield very similar results. The Plateau Updraft model presents an extra modeling degree-of-freedom. 


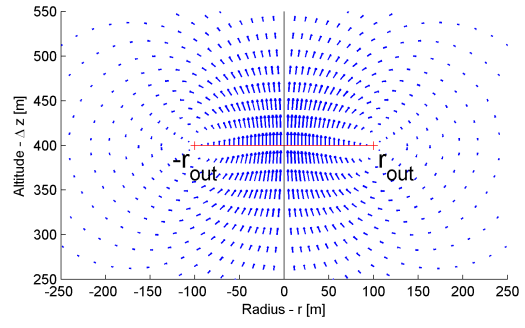

(a) 2D flow field

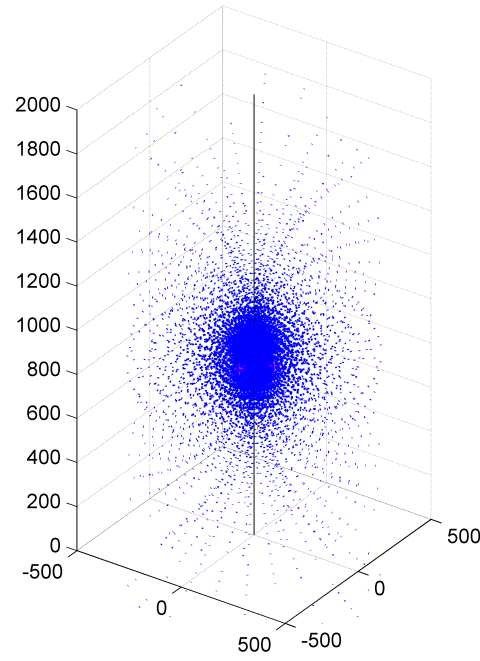

(b) 3D flow field

Figure 27: Flow field in a Bubble Thermal conservative model. The vortex is noticeable around the thermal updraft outer radius $(100 \mathrm{~m})$ at the mean altitude $(400 \mathrm{~m})$.

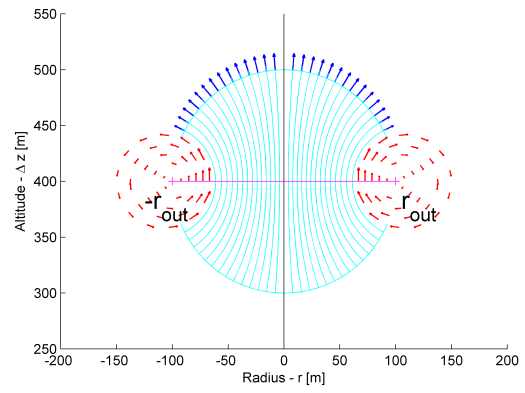

(a) 2D diagram

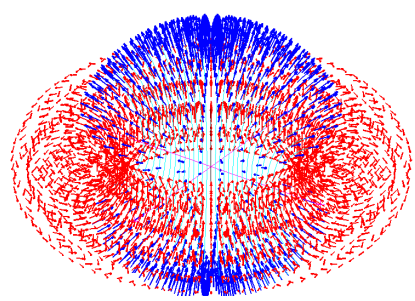

(b) 3D diagram

Figure 28: Bubble Thermal conservative model structure diagram. The vortex is illustrated by red arrows around the updraft outer radius. The blue arrows illustrate the updraft flow which birds and gliders usually take advantage of. 


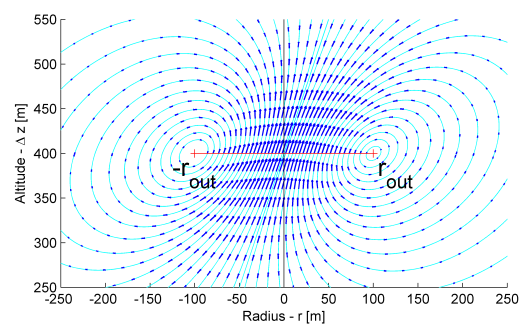

(a) 2D flow field

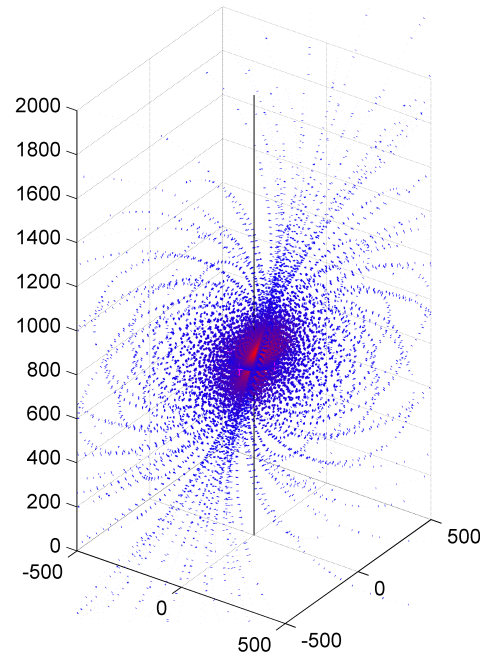

(b) 3D flow field

Figure 29: Flow field in a leaning Bubble Thermal. It is clear that the flow crosses the bubble center at a certain angle, which depends both on the bubble and the wind velocity.

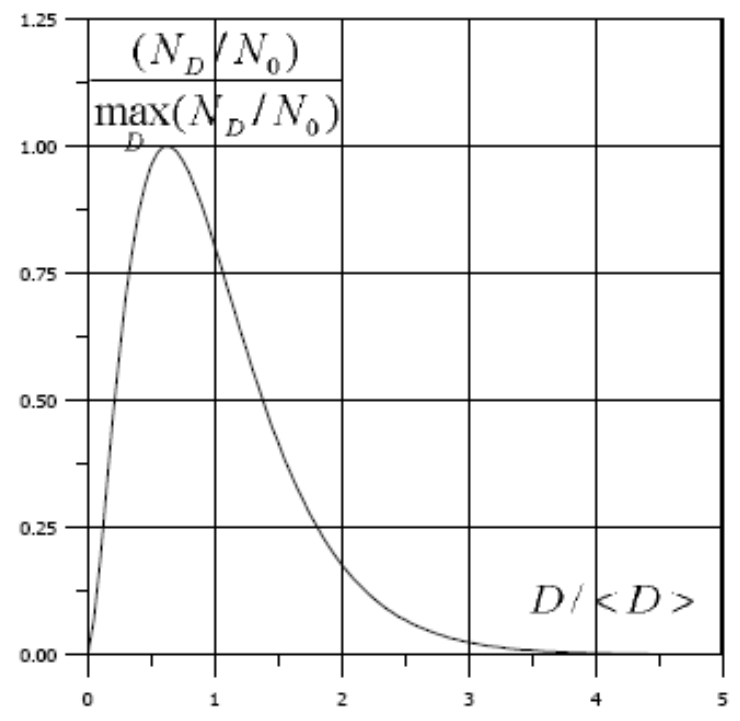

Figure 34: Diameter pdf with $a-1=5 / 3$ (illustrations from [22]) 


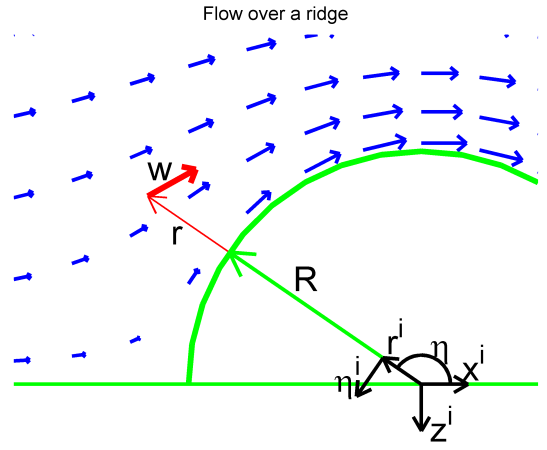

(a) Ridge flow approximation by a semicylindrical obstacle. In the picture $x^{i}, z^{i}, r^{i}$, and $\eta^{i}$ correspond to $\hat{\mathbf{x}}, \hat{\mathbf{z}}, \hat{\mathbf{r}}$, and $\hat{\eta}$, respectively.

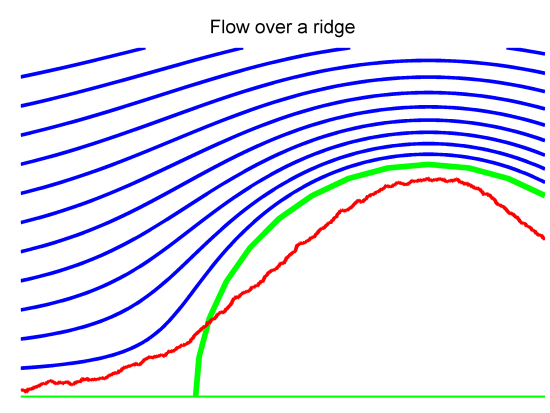

(b) Ridge flow stream-lines.

Figure 35: Ridge updraft flow field model.

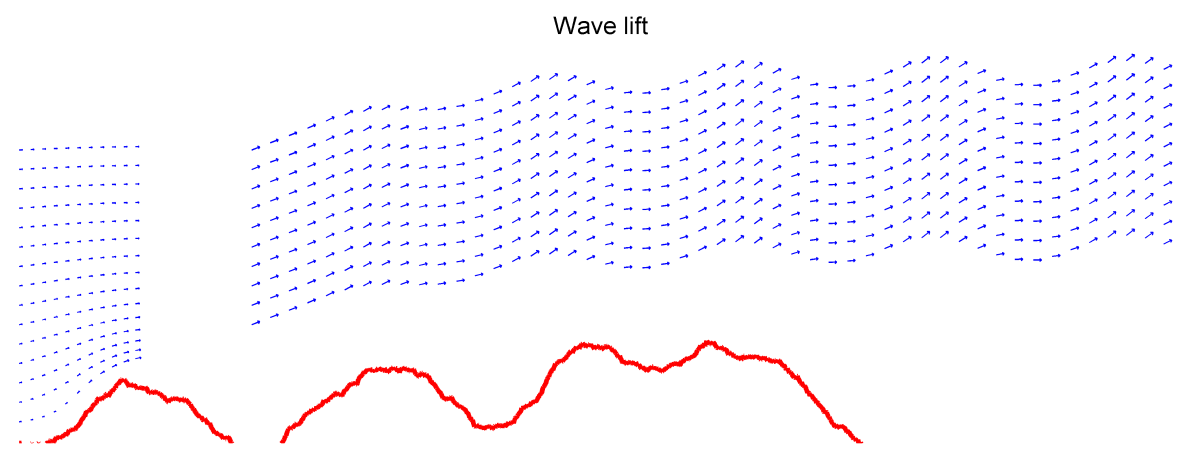

Figure 36: Wave flow. 